University of Nebraska - Lincoln

DigitalCommons@University of Nebraska - Lincoln

Papers in the Earth and Atmospheric Sciences

Earth and Atmospheric Sciences, Department

1995

\title{
Cretaceous Calcareous Plankton Biostratigraphy of Sites 872 through 879
}

\author{
Elisabetta Erba \\ Università degli Studi di Milano, elisabetta.erba@unimi.it \\ Isabella Premoli Silva \\ Università degli Studi di Milano \\ David K. Watkins \\ University of Nebraska-Lincoln, dwatkins1@unl.edu
}

Follow this and additional works at: https://digitalcommons.unl.edu/geosciencefacpub

Part of the Earth Sciences Commons

Erba, Elisabetta; Silva, Isabella Premoli; and Watkins, David K., "Cretaceous Calcareous Plankton Biostratigraphy of Sites 872 through 879" (1995). Papers in the Earth and Atmospheric Sciences. 220. https://digitalcommons.unl.edu/geosciencefacpub/220

This Article is brought to you for free and open access by the Earth and Atmospheric Sciences, Department of at DigitalCommons@University of Nebraska - Lincoln. It has been accepted for inclusion in Papers in the Earth and Atmospheric Sciences by an authorized administrator of DigitalCommons@University of Nebraska - Lincoln. 


\title{
8. CRETACEOUS CALCAREOUS PLANKTON BIOSTRATIGRAPHY OF SITES 872 THROUGH 879¹
}

\author{
Elisabetta Erba, ${ }^{2}$ Isabella Premoli Silva, ${ }^{2}$ and David K. Watkins ${ }^{3}$
}

\begin{abstract}
This paper documents the distribution of calcareous nannofossils and planktonic foraminifers in Cretaceous sequences recovered at Sites 872 through 879 drilled on guyots in the central and western Pacific Ocean during Leg 144. Calcareous plankton biostratigraphy allows us to obtain important biostratigraphic age constraints for the onset, development, and demise of shallow-water sedimentation on these guyots.

Site 872 on Lo-En Guyot is the only site at which shallow-water limestones were not recovered during Leg 144 . Here, the oldest sediment consists of pelagic limestone infilling fractures within the volcanic substrate. Calcareous plankton biostratigraphy constrains the age of these sediments to Coniacian to earliest Santonian (Dicarinella concavata planktonic foraminifer zone; CC14-CC15 nannofossil zones).

On Wodejebato Guyot (Sites 873 through 877), initiation of carbonate platform sedimentation occurred in the late Campanian (CC22 nannofossil Zone), continued during the late Campanian and Mastrichtian, and ended during the late, possibly latest, Maastrichtian. On MIT Guyot (Site 878), calcareous plankton biostratigraphy indicates that the initiation of carbonate platform sedimentation occurred in the earliest Aptian (lower part of the Chiastozygus litterarius nannofossil zone before the "nannoconid crisis"). After a phreato-magmatic eruption that deposited a polymictic breccia, carbonate sedimentation resumed in the middle late Aptian (Nannoconus truittii Acme, Globigerinelloides algerianus to Hedbergella trocoidea planktonic foraminifer zones). Shallow-water deposition ended during the late Albian after the Biticinella breggiensis Zone. Carbonate platform sedimentation at Site 879 on Takuyo-Daisan Guyot began during the middle late Aptian (N. truittii Acme, $G$. algerianus to $H$. trocoidea planktonic foraminifer zones) and ended by late Albian time (Rotalipora ticinensis planktonic foraminifer zone). Shallow-water limestone at Site 879 correlates with the upper carbonate platform sequence recovered at Site 878, but its thickness is only half that of the coeval sequence deposited on MIT Guyot, suggesting the presence of hiatuses.
\end{abstract}

\section{INTRODUCTION}

Leg 144 was devoted to the exploration of five guyots in the central and western Pacific Ocean (see site map preceding title page and Table 1) to reconstruct their geologic evolution and the age of inception and demise of the shallow-water carbonate sedimentation. Ten sites were occupied and 20 holes were drilled during the cruise (Premoli Silva, Haggerty, Rack, et al., 1993),

At all the guyots, volcanic substrate is represented by basalts that constructed emergent subaerial volcanic edifices that subsequently subsided to increasing water depths. Shallow-water limestone deposited over the volcanic substratum, and pelagic foraminifer and nannofossil ooze capped the guyots during the Tertiary and Quaternary (Premoli Silva, Haggerty, Rack, et al., 1993). Drilling on Lo-En Guyot indicated subaerial flows of late shield volcanism that were submerged before the late Turonian. Shallow-water limestone of Albian age was recovered on dredges from the southern flank of Lo-En Guyot (Lincoln et al., 1993), but it was not encountered at the drilled Site 872 (Premoli Silva, Haggerty, Rack, et al., 1993; ODP Leg 144 Shipboard Scientific Party, 1993).

The Cretaceous sedimentary sequences on the explored guyots are represented by shallow-water limestone. Calcareous nannofossils and planktonic foraminifers were extensively searched at all sites to obtain biostratigraphic ages based on calcareous plankton. We describe here the Cretaceous nannofossil and planktonic foraminifer assemblages recovered at Site 872 (Lo-En Guyot), Sites 873 through 877 (Wodejebato Guyot), Site 878 (MIT Guyot), and Site 879 (Takuyo-Daisan

'Haggerty, J.A., Premoli Silva, L., Rack, F., and McNutt, M.K. (Eds.), 1995. Proc ODP, Sci. Results, 144: College Station, TX (Ocean Drilling Program).

${ }^{2}$ Dipartimento di Scienze della Terra, Università degli Studi di Milano, Via L. Mangiagalli 34, 1-20133 Milano, Italy.

${ }^{3}$ Department of Geology, University of Nebraska, 330 Bessey Hall, Lincoln, NE 68588-0340, U.S.A.
Guyot) and discuss the biochronostratigraphic evidence for ages of inception, deposition, and demise of shallow-water limestones.

\section{MATERIAL AND METHODS}

Very detailed sampling was performed to ensure recovery of even sparse calcareous plankton in the shallow-water limestone (Table 2). The study was based on the examination of thin sections (both calcareous nannofossils and planktonic foraminifers), smear slides (calcareous nannofossils), washed residues, and cuttings (planktonic foraminifers). Smear slides were prepared using standard techniques: raw material was permanently mounted using the Norland Optical Adhesive. In addition, although some samples were ultrasonically cleaned and centrifuged to concentrate the extremely rare nannofossils, this procedure met with little success. Selected nannofossilbearing samples also were examined with the scanning electron microscope; however, the best results were derived from the study of smear slides and thin sections with a polarizing microscope. Semiquantitative and quantitative study of nannofossils was performed with a polarizing microscope, at $1250 \times$ magnification.

All thin sections prepared for sedimentological and paleontological analysis on board or for shore-based paleontological study were examined for planktonic foraminifers (see Premoli Silva et al. and Arnaud-Vanneau, both in this volume). This study was integrated with that based on isolated planktonic specimens, obtained from cuttings of poorly cemented limestone, collected by a sieve as each section of the core was split.

The results of this biostratigraphic study are reported separately for each guyot.

\section{SITE 872 (LO-EN GUYOT)}

The oldest sediments recovered at Site 872 occur in cracks within the volcanic substrate. Of the guyots explored during Leg 144, Lo-En is the only one where shallow-water limestone was not recovered at the drill site. Here, in fact, pelagic limestone immediately overlies the 
Table 1. Latitude, longitude, and water depth for Sites 872 through 879.

\begin{tabular}{lllll}
\hline Site & \multicolumn{1}{c}{ Guyot } & $\begin{array}{c}\text { Latitude } \\
(\mathrm{N})\end{array}$ & $\begin{array}{c}\text { Longitude } \\
\text { (E) }\end{array}$ & $\begin{array}{c}\text { Water depth } \\
\text { (mbsf) }\end{array}$ \\
\hline 872 & Lo-En & $10^{\circ} 05.85^{\prime}$ & $162^{\circ} 51.96^{\prime}$ & 1084 \\
873 & Wodejebato & $11^{\circ} 53.84^{\prime}$ & $164^{\circ} 55.20^{\prime}$ & 1334 \\
874 & Wodejebato & $12^{\circ} 00.76^{\prime}$ & $164^{\circ} 55.22^{\prime}$ & 1374 \\
875 & Wodejebato & $12^{\circ} 00.72^{\prime}$ & $164^{\circ} 56.44^{\prime}$ & 1409 \\
876 & Wodejebato & $12^{\circ} 01.476^{\prime}$ & $164^{\circ} 55.90^{\prime}$ & 1399 \\
877 & Wodejebato & $12^{\circ} 01.14^{\prime}$ & $164^{\circ} 55.30^{\prime}$ & 1355 \\
878 & MIT & $27^{\circ} 19.143^{\prime}$ & $151^{\circ} 53.028^{\prime}$ & 1323 \\
879 & Takuyo-Daisan & $34^{\circ} 10.46^{\prime}$ & $144^{\circ} 18.56^{\circ}$ & 1501 \\
\hline
\end{tabular}

basalt and is followed by a volcanic pebble conglomerate, and foraminifer-nannofossil ooze that capped the guyot during the Tertiary and Quaternary. Calcareous nannofossil and planktonic foraminifers from the cracks in the volcanic substrate constrain the age of the oldest sediments at this site.

\section{Calcareous Nannofossils}

A few layers with moderately to poorly preserved Cretaceous nannofossils were detected in Core $144-872 \mathrm{~A}-17 \mathrm{H}$, which penetrated the contact between the pelagic limestone and the underlying basalt, and in Core 144-872B-4R, which recovered basalts with thin interbasalt sediment layers (Premoli Silva, Haggerty, Rack et al., 1993). Sample 144-872A-17H-CC yielded a nannofossil assemblage containing Micula concava, Reinhardtites anthophorus, Aspidolithus parcus expansus, Arkangelskiella specillata, Stoverius biarcus, Stoverius coronatus, and Gephyrorhabdus coronadventis (Table 3 ). This nannoflora is correlated with Santonian Zones CC15-17 of Sissingh (1977) (Fig. 1).

In Core 144-872B-4R, five sediment layers yielded common and moderately well preserved calcareous nannofossils (Table 3 ). The cooccurrence of Lithastrinus septenarius, Reinhardtites anthophorus, Micula decussata, and Eiffellithus eximius in Sample 144-872B-4R$1,21-22 \mathrm{~cm}$, indicates Zone CC15 of Sissingh (1977). Sample 144872 B-4R-1, 60-62 cm, contains frequent specimens of Nannoconus farinacciae along with Lithastrinus septenarius and, therefore, is correlated with the late Coniacian to early Santonian Zones CC14 and CC15 of Sissingh (1977) (Fig. 1). Only sparse specimens of Eiffellithus eximius were observed in Sample 144-872B-4R-1, 68-89 cm, which cannot be older than early Turonian based on nannofossil biostratigraphy. However, planktonic foraminifers from this interval indicate the Dicarinella concavata Zone of Coniacian to earliest Santonian age.

\section{Planktonic Foraminifers}

Planktonic foraminifers co-occur with calcareous nannofossils in Hole $872 \mathrm{~B}$ samples but are absent in Hole $872 \mathrm{~A}$. In the lower samples (144-872B-4R-1, 46-48 cm; 144-872B-4R-1, 60-62 cm, and 144-872B-4R-1, 68-71 cm), planktonic foraminifer assemblages are characterized by abundant marginotruncanids (e.g., M. sigali, $M$. schneegansi, M. pseudolinneiana, M. coronata) and Heterohelix reussi, common Dicarinella concavata, and rare whiteinellids and Dicarinella primitiva. Rare, older forms possibly attributable to Dicarinella hagni and Marginotruncana marianosi are recorded in Sample 144-872B-4R-1, 68-71 cm. The bulk of the planktonic faunas in all three layers is indicative of the Dicarinella concavata Zone. According to the revised calibration of Premoli Silva and Sliter (1994; see also below), this zone spans the interval from upper Turonian to lowermost Santonian; however, the most probable age for these sediments is Coniacian. The possible older forms, D. hagni and M. marianosi, become extinct in the late middle Turonian (Marginotruncana sigali Zone). Poor recovery and the discontinuous record make it difficult to determine whether the older aspect of the assemblage is related to reworking or to preservation. There is evidence in favor of both hypotheses. On one hand, the occurrence of shallow-water foraminifers and other skeletal organisms within the faunal-rich pelagic sediments would support the possibility of some transport and reworking. On the other hand, owing to the poor preservation and high degree of phosphatization in the lowermost sample the older species mentioned above may be misidentified.

The topmost sample (Sample 144-872B-4R-1, 18-22 cm) yields common to abundant, rather well-preserved planktonic foraminifers. The assemblage, substantially similar to that from the previous samples, includes common marginotruncanids belonging to the same species as identified in the underlying assemblages, several Dicarinella concavata, Globigerinelloides bollii, Heterohelix reussi, and rare Contusotruncana fornicata, Archaeoglobigerina cretacea, Globotruncana linneiana, Ventilabrella eggeri, and V. glabrata. This assemblage indicates the Dicarinella asymetrica Zone, even in the absence of the nominate species and is probably early Santonian in age.

Other notable features of this sequence are the occurrence of several medium-sized, perfectly preserved fish teeth and abundant fecal pellets (Favreina sp.), and a few benthic foraminifers indicative of rather shallow-water environment in the lowermost samples.

Two species of benthic foraminifers associated with calcareous plankton are Dictyopselloides sp. (Samples 144-872B-4R-1, 46-48, 60-62, and 68-71 cm) and Dorothia sp. cf. D. bulletta (Sample 144$872 \mathrm{~B}-4 \mathrm{R}-1,68-71 \mathrm{~cm})$. The latter species is known from the Upper Cretaceous, whereas the genus Dictyopselloides is known from the Santonian of France and Spain. Their occurrence is consistent with that inferred from calcareous plankton.

These benthic foraminifers, with a bathymetric range from upper to middle neritic (maximum paleodepth close to $200 \mathrm{~m}$ ), suggest a relatively shallow paleodepth for the pelagic fracture infillings, although the possibility of post-mortem transport or reworking cannot be ruled out.

In summary, the oldest sediment preserved on Lo-En Guyot consists of Coniacian to early Santonian pelagic limestone occurring as fracture infillings and volcanic pebble conglomeratic matrix lying atop basaltic substrate. Planktonic foraminifers and, to a lesser extent, calcareous nannofossils constrain the age of this material as Coniacian to early Santonian. However, sediments as old as late middle Turonian may have been deposited on the guyot. The presence of middle neritic benthic foraminifers in the lowest deposits may indicate either a relatively shallow pelagic paleoenvironment or the transport of shallowwater material into a deeper site of deposition. The Coniacian to early Santonian fossils are extensively phosphatized, indicating an extended period of exposure on the seafloor.

\section{SITES 873 THROUGH 877 (WODEJEBATO GUYOT)}

Wodejebato Guyot formed during the Cretaceous as a volcanic edifice and platform sediments were deposited during the Late Cretaceous. The demise of the shallow-water limestone occurred during the Maastrichtian, and pelagic foraminifer and nannofossil oozes capped the guyot during the Tertiary and Quaternary (Premoli Silva, Haggerty, Rack, et al., 1993). The platform limestone of the Cretaceous sedimentary sequence at Sites 873 through 877 was extensively searched for calcareous nannofossils and planktonic foraminifers to obtain biostratigraphic ages and calibration to zonal schemes based on calcareous plankton. Calcareous nannofossils were not observed in the platform sequences recovered at Sites 875 and 876 on the outer ridge, whereas a few nannofossiliferous layers were found at the base of the shallow-water limestones at Sites 873, 874, and 877.

Planktonic foraminifers were observed at all site, with increasing abundance in both persistence of occurrence and species richness per sample, from the lagoonal Site 873 to the inner ridge Sites 874 and 877. Maximum abundance and diversity occur at the outer ridge Sites 875 and 876. 


\section{LATE CRETACEOUS}

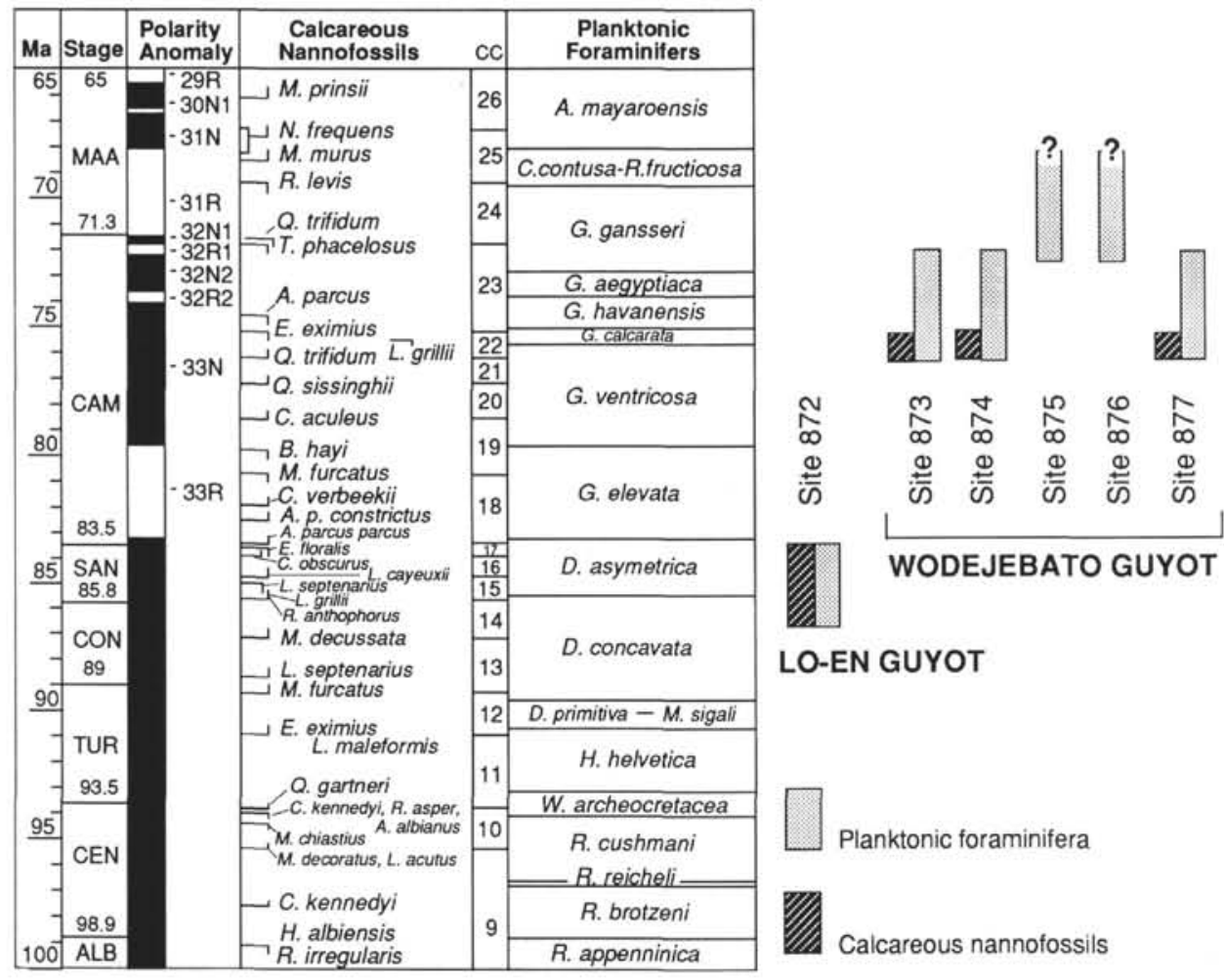

Figure 1. Updated time scale (Gradstein et al., 1994) and revised biomagnetostratigraphy for the Late Cretaceous. Biostratigraphic ages at Sites 872 through 877 are based on the occurrence of calcareous nannofossils and planktonic foraminifers in shallow-water limestone from Wodejebato Guyot (Sites 873 through 877 ) and in the oldest sediments infilling fractures within the volcanic substrate at Lo-En Guyot (Site 872). Age scale after Gradstein et al. (1994); nannofossil zonation of Sissingh (1977); planktonic foraminifer zonation of Premoli Silva and Sliter (1994).

\section{Calcareous Nannofossils}

Nannofossils are well preserved and highly diverse (= number of species) in the basal sediments at Sites 873,874 , and 877 , but abundances are extremely low. A detailed documentation including quantitative distribution of nannofloras from Wodejebato Guyot is given by Erba et al. (this volume). Our remarks here concern only the biostratigraphy (Fig. 1). Calcareous nannofossils occur only at the base of the platform sequence at Sites 873,874 , and 877 and date the onset of marine sedimentation. At Site 877 , the occurrence of the marker species Quadrum trifidum, Eiffellithus eximius, and Reinhardtites anthophorus indicates the CC22 Zone (Sissingh, 1977) of late Campanian age. At Sites 873 and 874 nannofloral assemblages indicate a broad Campanian age, but key species with a more restricted range were not observed. However, the volcanic pedestal at all sites drilled on Wodejebato Guyot consists of magnetically reversed basalts attributed to magnetic polarity Chron 33R of early Campanian age (Premoli Silva, Haggerty, Rack, et al., 1993; Nakanishi, Gee, et al., 1992). Therefore, the oldest marine sediments at Sites 873 and 874 are datable as late Campanian. Because the nannofloral composition is very similar at Sites 873,874 , and 877 , we also attribute the oldest marine sediments of Sites 873 and 874 to the CC22 Zone of Sissingh (1977) (Fig. 1).

The species Corollithion kennedyi, which is restricted to the Cenomanian was found at Site 877 suggesting the presence of older sediments elsewhere on Wodejebato Guyot. Other pre-Campanian species include Axopodorhabdus albianus, Eprolithus floralis, and Rhagodiscus asper. Evidence of reworking also is given by the occurrence of recrystallized, broken, and often phosphatized specimens of long-ranging taxa such as Watznaueria barnesae, Cretarhabdus surirellus, and Zygodiscus erectus (see Erba et al., this volume).
Several samples analyzed from the remainder of the platform limestone at Sites 873, 874, and 877, were barren of calcareous nannofossils.

\section{Planktonic Foraminifers}

The planktonic foraminifer assemblages are, in general, poorly diversified as was expected in such shallow-water depths of Wodejebato carbonate platform. The most consistent forms of the generally small-sized faunas are Archaeoglobigerina, and secondarily Heterohelix and Globigerinelloides. Keeled forms occur sporadically. The spatial and temporal distribution of planktonic foraminifers shows a clear trend. Keeled forms, known to be deeper dwelling inhabitants than the globigeriniforms (Caron and Homewood, 1983), are more abundant in the outer ridge sites. In addition, keeled forms occur in the uppermost part of the carbonate sequence at the other three sites (see Tables 4-8). Most of the identified taxa are long-ranging species, but the keeled group contains the most age-diagnostic species found on Wodejebato; the Gansserina gansseri group. The nominate taxon identifies the $G$. gansseri Zone, which according to the revised calibration (see Chronostratigraphy below) straddles the Campanian/ Maastrichtian boundary and extends through the middle Maastrichtian. Representatives of the Gansserina gansseri group (e.g., G. gansseri and $G$. wiedenmayeri) occur at the top of the carbonate sequence at Sites 873 and 877 (Tables 4 and 8 ), one core above the base of the limestone unit at Site 875, and almost at the base at Site 876 (Tables 6 and 7). On the other hand, this group was not found at Site 874, which yielded the poorest and fewest planktonic assemblages (Table 5) (see also Premoli Silva et al., this volume). These occurrences suggest the top of the carbonate sequence at Sites 873 and 877 , and 
Table 2. Samples investigated for calcareous nannofossils listing the type of investigation, key species, and biozones.

\begin{tabular}{|c|c|c|c|c|c|c|c|c|c|}
\hline $\begin{array}{l}\text { Core, section. } \\
\text { interval }(\mathrm{cm})\end{array}$ & $\begin{array}{l}\text { Smear } \\
\text { slide }\end{array}$ & $\begin{array}{l}\text { Thin } \\
\text { section }\end{array}$ & SEM & Nannofossils & $\begin{array}{l}\text { Core, section, } \\
\text { interval }(\mathrm{cm})\end{array}$ & $\begin{array}{l}\text { Smear } \\
\text { slide }\end{array}$ & $\begin{array}{l}\text { Thin } \\
\text { section }\end{array}$ & SEM & Nannofossils \\
\hline Lo-En Guyot & & & & & IR-CC, 13-15 & & $\mathrm{X}$ & & - \\
\hline $144-872 \mathrm{~A}=$ & & & & & $2 \mathrm{R}-1.20-23$ & & $\mathrm{x}$ & & late middle Albian \\
\hline $17 \mathrm{H}-\mathrm{CC}$ & & $\mathrm{x}$ & & $\mathrm{CC} 15-17$ & $3 R-1,40-46$ & & $\mathrm{X}$ & & late middle Albian \\
\hline 144-872B- & & & & & 15R-CC & $\mathrm{X}$ & & & - \\
\hline $4 \mathrm{R}-1,0-1$ & $\mathrm{X}$ & & & - & $36 \mathrm{M}-1.98-105$ & $\mathrm{X}$ & & & - \\
\hline $4 \mathrm{R}-1,20-21$ & $\mathrm{X}$ & & & $\overline{\mathrm{CC} 15}$ & $41 \mathrm{M}-1,0-4$ & & $\underset{X}{X}$ & & N. truittii Acme \\
\hline $4 \mathrm{R}-1,21-22$ & $\mathrm{X}$ & & & CCI5 & & $\mathrm{x}$ & $\mathrm{X}$ & & N. truittii Acme \\
\hline $4 \mathrm{R}-1,60-62$ & & $\mathrm{X}$ & & $\mathrm{CCl} 4-15$ & $\begin{array}{l}42 \mathrm{M}-1,5-10 \\
42 \mathrm{M}-1,15-20\end{array}$ & & $\mathrm{X}$ & & - \\
\hline $4 \mathrm{R}-1,61-62$ & $\underset{\mathrm{X}}{\mathrm{X}}$ & & & E. eximius $+M$. furcatus & $42 \mathrm{M}-1,22-25$ & & $\hat{x}$ & & E \\
\hline $4 \mathrm{R}-1,68-89$ & $\begin{array}{l}X \\
x\end{array}$ & & & E. eximius & $44 \mathrm{M}-\mathrm{CC}$ & $\mathrm{X}$ & & & - \\
\hline $\begin{array}{l}5 R-1,127-128 \\
5 R-2,12-20\end{array}$ & $\begin{array}{l}X \\
X\end{array}$ & & & - & $45 \mathrm{M}-\mathrm{CC}$ & $\mathrm{x}$ & & & - \\
\hline $\begin{array}{l}5 R-2,12-20 \\
5 R-2,44-47\end{array}$ & $\begin{array}{l}X \\
X\end{array}$ & & & 一 & $50 \mathrm{R}-3$ (base) & $\mathrm{X}$ & & & - \\
\hline $5 R-2,96-97$ & $\hat{x}$ & & & - & $51 R-2,5-10$ & & $\mathrm{X}$ & & - \\
\hline $5 \mathrm{R}-2,122-124$ & $\mathrm{x}$ & & & - & $52 \mathrm{R}-\mathrm{CC}$ & $\mathrm{X}$ & & & - \\
\hline $5 R-4,25-27$ & $\mathrm{x}$ & & & - & 53R-CC & $\mathrm{X}$ & & & - \\
\hline Woejebato Guyot & & & & & $\begin{array}{l}63 R-2,2-4 \\
62 R-2,112-113\end{array}$ & $\begin{array}{l}x \\
x\end{array}$ & & & - \\
\hline $144-873 \mathrm{~A}-$ & & & & & $62 \mathrm{R}-2,120$ & $\hat{\mathrm{X}}$ & & & - \\
\hline 2R-CC & $\mathrm{X}$ & & & - & $62 \mathrm{R}-2,130$ & $\mathrm{X}$ & & & - \\
\hline $3 \mathrm{R}-\mathrm{CC}$ & $\mathrm{x}$ & & & - & $62 \mathrm{R}-2,140$ & $\mathrm{X}$ & & & - \\
\hline $4 \mathrm{R}-\mathrm{CC}$ & $\mathrm{x}$ & & & - & $63 \mathrm{R}-1,0-1$ & $\mathrm{X}$ & & & - \\
\hline $5 \mathrm{R}-\mathrm{CC}$ & $\mathrm{x}$ & & & - & $63 \mathrm{R}-1,6-8$ & $\mathrm{X}$ & & & 一 \\
\hline $6 \mathrm{R}-\mathrm{CC}$ & $\mathrm{x}$ & & & - & $63 \mathrm{R}-1,10-11$ & $\mathrm{X}$ & & & - \\
\hline $7 \mathrm{R}-\mathrm{CC}$ & $\mathrm{X}$ & & & - & $75 \mathrm{R}-1,141-143$ & & $\mathrm{X}$ & & lower $C$. litterarius Zone \\
\hline $8 \mathrm{R}-1,110-116$ & $\mathrm{X}$ & & & - & $75 \mathrm{R}-2,0-4$ & & $\mathrm{X}$ & & lower $C$. litterarius Zone \\
\hline $8 R-2,5-8$ & $X$ & & & - & $77 \mathrm{R}-1,28-31$ & & $\mathrm{x}$ & & W. barnesae $+R$. irregularis \\
\hline $8 R-2,9-12$ & $\mathrm{x}$ & & & Prediscosphaera sp. & $79 \mathrm{R}-5,80-81$ & $x$ & & & - \\
\hline $9 \mathrm{R}-2,16-22$ & $\mathrm{x}$ & & & - & $79 \mathrm{R}-5,100-105$ & $\mathrm{X}$ & & & - \\
\hline $11 R-2,15-16$ & $\mathrm{X}$ & & & - & $94 \mathrm{R}-5,0-1$ & $\mathrm{X}$ & & & - \\
\hline IIR-2, 20 & $\mathrm{X}$ & & $\mathrm{X}$ & Campanian & Takuyo-Daisan Guyot & & & & \\
\hline $\begin{array}{l}\text { IIR-2, 17-22 } \\
\text { 12R-CC }\end{array}$ & $\underset{\mathrm{X}}{\mathrm{X}}$ & & $\mathrm{X}$ & Campanian & $144-879 \mathrm{~A}-$ & & & & \\
\hline $12 \mathrm{R}-\mathrm{CC}$ & $\mathrm{X}$ & & & - & $2 \mathrm{R}-1.32-38$ & & $\mathrm{X}$ & & - \\
\hline 144-873B- & & & & & $2 \mathrm{R}-1,132-138$ & & $\mathrm{X}$ & & - \\
\hline $9 \mathrm{~N}-\mathrm{CC}$ & $X$ & & & - & $5 R-1,0-2$ & $\mathrm{X}$ & & & - \\
\hline $1 \mathrm{~N}-\mathrm{CC}$ & $\mathrm{X}$ & & & - & $15 R-1,0-3$ & & $\mathrm{X}$ & & - \\
\hline $144-874 B-$ & & & & & $16 \mathrm{R}-1,12-13$ & $\mathrm{X}$ & & & 二 \\
\hline $21 \mathrm{R}-1,0-1$ & $\mathrm{X}$ & & & - & $\begin{array}{l}16 \mathrm{R}-1,14-15 \\
16 \mathrm{R}-1,17-18\end{array}$ & $\underset{x}{X}$ & & & - \\
\hline 2 IR-1, $35-36$ & $\mathrm{X}$ & & & - & $\begin{array}{l}16 \mathrm{R}-1,17-18 \\
16 \mathrm{R}-1,18-20\end{array}$ & $\stackrel{\lambda}{x}$ & & & - \\
\hline $2 \mid \mathrm{R}-1,40-41$ & $\mathrm{X}$ & & $\mathrm{X}$ & Campanian & $16 \mathrm{R}-1,23-27$ & & $\mathrm{X}$ & & - \\
\hline $21 \mathrm{R}-1,44-45$ & $\mathrm{X}$ & & & - & $16 \mathrm{R}-1,25-26$ & $\mathrm{X}$ & & & N. truittii Acme \\
\hline $21 \mathrm{R}-1,148-149$ & $\mathrm{X}$ & & & 二 & $16 \mathrm{R}-1,27-33$ & & $\mathrm{X}$ & & N. truittii Acme \\
\hline 21R-CC & $\mathrm{X}$ & & & - & $16 \mathrm{R}-1,37-38$ & $\mathrm{x}$ & & & - \\
\hline $144-875 \mathrm{C}-$ & & & & & $16 \mathrm{R}-1,44-45$ & $\mathrm{X}$ & & & - \\
\hline $8 \mathrm{M}-\mathrm{CC}$ & $\mathrm{x}$ & & & - & $16 \mathrm{R}-1,60-63$ & $\mathrm{X}$ & & & - \\
\hline $10 \mathrm{M}-\mathrm{CC}$ & $\mathrm{X}$ & & & - & $16 \mathrm{R}-1,68-72$ & $\mathrm{X}$ & & & N. truittii Acme \\
\hline $11 \mathrm{M}-\mathrm{CC}$ & $\mathrm{X}$ & & & - & $16 \mathrm{R}-1,74-77$ & $\mathrm{X}$ & & & - \\
\hline $12 \mathrm{M}-\mathrm{CC}$ & $\mathrm{x}$ & & & - & $16 \mathrm{R}-1,79-84$ & & $\mathrm{X}$ & & - \\
\hline $13 \mathrm{M}-\mathrm{CC}$ & $\mathrm{x}$ & & & - & $16 \mathrm{R}-1,94-96$ & $\mathrm{X}$ & & & - \\
\hline $144-876 \mathrm{~A}-$ & & & & & $\begin{array}{l}16 \mathrm{R}-1,102-103 \\
16 \mathrm{R}-1,123-125\end{array}$ & $\mathrm{X}$ & $\mathrm{x}$ & & - \\
\hline IIR-2, 13 & $\mathrm{X}$ & & & - & $16 \mathrm{R}-1,137-145$ & $\mathrm{x}$ & $\lambda$ & & $\overline{-}$ \\
\hline IIR-2, 43-44 & $\mathrm{X}$ & & & - & $17 R-1,0-5$ & & $\mathrm{X}$ & & - \\
\hline $11 \mathrm{R}-2,56-62$ & $\mathrm{X}$ & & & - & $17 \mathrm{R}-1,18-19$ & $\mathrm{x}$ & & & - \\
\hline $14 R-1,0-1$ & $\mathrm{X}$ & & & - & $17 \mathrm{R}-1,23-24$ & $\mathrm{X}$ & & & - \\
\hline $144-877 \mathrm{~A}-$ & & & & & $17 \mathrm{R}-1,24-25$ & $\mathrm{X}$ & & & - \\
\hline IR-1. 140-144 & $\mathrm{x}$ & & & - & $17 \mathrm{R}-1,29-30$ & $\mathrm{x}$ & & & - \\
\hline IR-1, 144-148 & $\mathrm{x}$ & & & - & $17 \mathrm{R}-1.36-37$ & $\mathrm{X}$ & & & - \\
\hline $3 \mathrm{R}-1,29-30$ & $x$ & & & - & $17 \mathrm{R}-1.50-51$ & $X$ & & & - \\
\hline $3 \mathrm{R}-1,93-94$ & $\mathrm{x}$ & & & - & $17 R-1,57-61$ & & $X$ & & - \\
\hline 7R-CC & $x$ & & & - & $17 \mathrm{R}-1,70-80$ & $\mathrm{X}$ & $\mathrm{X}$ & $\mathrm{X}$ & N. truittii Acme \\
\hline $14 \mathrm{R}-\mathrm{CC}$ & $\mathrm{x}$ & & & - & $17 \mathrm{R}-1.73-74$ & $\mathrm{X}$ & & & N. truittii Acme \\
\hline $16 \mathrm{R}-\mathrm{CC}$ & $\mathrm{X}$ & & & - & $17 \mathrm{R}-1,78-79$ & $\mathrm{X}$ & & & W. barnesae \\
\hline 18R-CC & $\mathrm{X}$ & & & - & $17 \mathrm{R}-1,79-80$ & $\mathrm{X}$ & & & - \\
\hline 19R-CC & $\mathrm{x}$ & & & - & $17 \mathrm{R}-1,80-83$ & $\mathrm{X}$ & $\mathrm{X}$ & $\mathrm{X}$ & N. truittii Acme \\
\hline $20 \mathrm{R}-1,16-17$ & $x$ & & $\mathrm{x}$ & Campanian & $17 \mathrm{R}-1,83-85$ & $\mathrm{X}$ & & & - \\
\hline $20 \mathrm{R}-1,28-29$ & $\mathrm{x}$ & & & - & $17 R-1,95-97$ & $\mathrm{X}$ & & & - \\
\hline $20 \mathrm{R}-1,42-44$ & $\mathrm{x}$ & & $\mathrm{x}$ & Campanian & $17 \mathrm{R}-1,97-103$ & $\mathrm{X}$ & $\mathrm{X}$ & $\mathrm{X}$ & N. truittii Acme \\
\hline $20 \mathrm{R}-1,44-45$ & $\mathrm{X}$ & & $\mathrm{x}$ & $\mathrm{CC} 22$ & $17 \mathrm{R}-1,109-112$ & & $\mathrm{X}$ & & - \\
\hline $20 \mathrm{R}-1,54-55$ & $\mathrm{x}$ & & & - & I $7 \mathrm{R}-1,119-120$ & $X$ & & & - \\
\hline $20 \mathrm{R}-1,70-71$ & $\mathrm{x}$ & & $\mathrm{x}$ & Late Cretaceous & $17 R-1,133-134$ & $\mathrm{X}$ & & & - \\
\hline $20 \mathrm{R}-1,81-82$ & $\mathrm{x}$ & & & - & $17 \mathrm{R}-1,146-147$ & $X$ & & & - \\
\hline $20 \mathrm{R}-1,92-94$ & $\mathrm{x}$ & & & - & $17 \mathrm{R}-2,25-26$ & $X$ & & & R. irregularis $+\mathrm{Z}$ erectus \\
\hline $20 \mathrm{R}-1,101-102$ & $\mathrm{x}$ & & & - & $17 \mathrm{R}-2,40-41$ & $X$ & & & - \\
\hline $20 \mathrm{R}-1,102-103$ & $\mathrm{X}$ & & & - & $17 R-2,55-56$ & $\mathrm{X}$ & & & - \\
\hline $20 \mathrm{R}-2,0-1$ & $\hat{\mathrm{x}}$ & & & - & 17R-CC & $\mathrm{X}$ & & & - \\
\hline $20 \mathrm{R}-2,1-2$ & $\mathrm{x}$ & & & - & $18 \mathrm{R}-1,4-7$ & & $\mathrm{X}$ & & - \\
\hline $20 \mathrm{R}-2,15-16$ & $x$ & & & - & $18 \mathrm{R}-1,10-13$ & & $\mathrm{X}$ & & - \\
\hline $20 \mathrm{R}-2,34-35$ & $\mathrm{x}$ & & & - & $18 \mathrm{R}-1,22-26$ & & $\mathrm{X}$ & & - \\
\hline $20 \mathrm{R}-2,61-62$ & $\mathrm{X}$ & & & - & $18 \mathrm{R}-1,23-24$ & $\mathrm{X}$ & & & - \\
\hline $20 \mathrm{R}-2,98-99$ & $\mathrm{x}$ & & & - & I8R-1, $30-35$ & $\mathrm{X}$ & & & N. truittii Acme \\
\hline MIT Guyot & & & & & 18R-1, 31-37 & $\mathrm{X}$ & $\mathrm{X}$ & $\mathrm{X}$ & late Aptian \\
\hline 144-878Ä- & & & & & $21 k-1,95-90$ & & & & \\
\hline
\end{tabular}


Table 3. Range chart of calcareous nannofossils in the oldest marine sediments recovered at Site 872 (Lo-En Guyot).

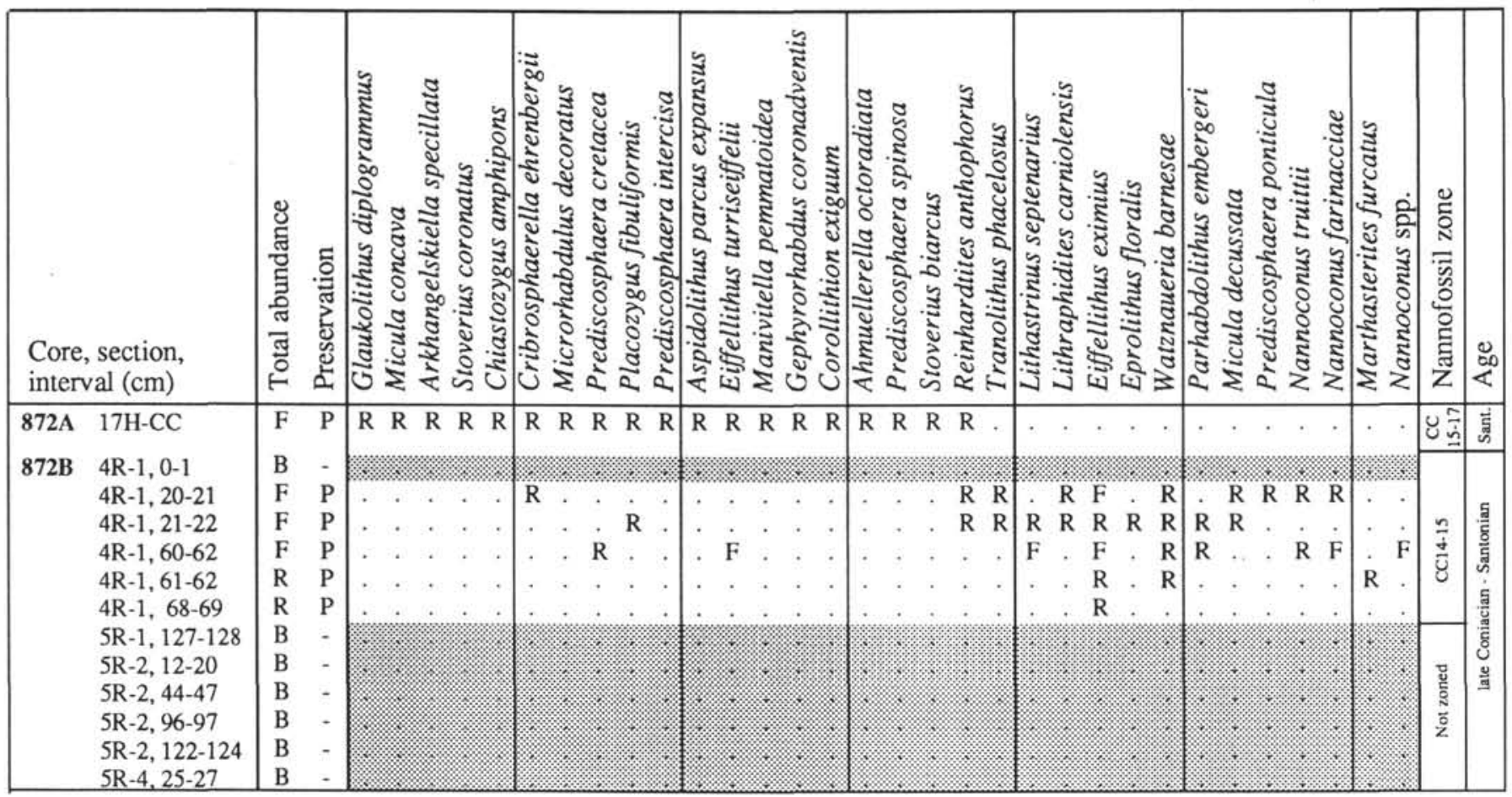

Notes: Shaded areas are barren of nannofossils. Estimates of the total nannofossil abundance are given as $\mathrm{F}$ (few) $=0.1 \%-1 \%$ of all particles, R (rare) $=<0.1 \%$ of all particles, and B (barren) $=$ no nannofossils observed. Estimates of preservation are given as $\mathrm{P}($ poor $)=$ most specimens display significant amounts of overgrowth/dissolution, and species identification is sometimes impaired. Estimates of the relative abundance of nannofossil species are given as $F(f e w)=$ one specimen per $2-10$ fields of view, and $R$ (rare) $=$ one specimen per $11-100$ fields of view.

possibly at Site 874 , correlates with the base of the carbonate sequence at Site 876 and with the lower part of the sequence at Site 875 . Consequently, most of the carbonate succession recovered at these latter sites is younger than that drilled in the lagoonal and outer ridge locations. The occurrence of benthic foraminifers is consistent with this assumption (see Premoli Silva et al., this volume).

At Site 876, G. wiedenmayeri is still present at the top of the sequence. This indicates that most, if not all, of the carbonate succession at this site is Maastrichtian and is apparently the most complete record of the upper part of the carbonate sequence at Wodejebato Guyot (see also Premoli Silva et al., this volume). The absence of Abathomphalus mayaroensis, the uppermost Maastrichtian zonal index, or alternatively of Racemiguembelina fructicosa and Contusotruncana contusa, two species appearing just below the first occurrence (FO) of $A$. mayaroensis, precludes a precise age determination for the uppermost limestone. On the other hand, the presence of several species of rugoglobigerinids in the upper portion of the less complete carbonate succession at Site 875 indicates that the platform continued to develop during the late Maastrichtian at the outer ridge sites. This assumption is corroborated by the occurrence of common Maastrichtian planktonic foraminifers from sedimentary infillings of cavities in the uppermost platform limestone at the inner ridge Site 874 (144-874B-3R-1, $114-118 \mathrm{~cm})$. The planktonic fauna from the infillings contains common Hedbergella monmouthesis and Heterohelix globulosa along with other very rare species including a possible Globotruncanella. This assemblage indicates a Maastrichtian age that may be as young as late Maastrichtian. Therefore, we conclude that the carbonate platform deposition ceased before the end of the Cretaceous.

In summary, calcareous nannofossils and planktonic foraminifers indicate that (1) the initiation of carbonate platform sedimentation occurred in the late Campanian (CC22 Zone of Sissingh, 1977), (2) carbonate sedimentation continued during the late Campanian and Maastrichtian, and (3) carbonate sedimentation ended during the late Maastrichtian (possibly pre-A. mayaroensis Zone).

\section{SITE 878 (MIT GUYOT)}

At Site 878 a very thin pelagic cap overlies $700 \mathrm{~m}$ of shallowwater sediments that were deposited over a volcanic edifice during the Aptian-Albian (Premoli Silva, Haggerty, Rack, et al., 1993). Only a few samples yielded biostratigraphically useful calcareous plankton, despite extensive searching.

\section{Calcareous Nannofossils}

Several samples from the shallow-water limestone sequence at Site 878 (MIT Guyot) were analyzed for nannofossils, but very few yielded nannofloras (Table 9). The oldest nannofossil-bearing sample (Sample 144-878A-77R-1, 28-31 cm) contained sparse specimens of the long-ranging species Watznaueria barnesae along with Rucinolithus irregularis. The latter taxon indicates that the base of the lower shallow-water sequence cannot be older than latest Barremian (Coccioni et al., 1992). A more abundant and diversified nannoflora from Samples 144- 878A-75R-2, 0-4 cm, and -75R-1, 141-143 cm, includes Watznaueria barnesae, Rucinolithus irregularis, Rucinolithus terebrodentarius, Rhagodiscus asper, Nannoconus colomii, Nannoconus st. steinmannii, Nannoconus bucheri, Nannoconus wassallii, Nannoconus circularis, Nannoconus globulus, Nannoconus truittii, Cyclagelosphaera margerelii, and Parhabdolithus embergeri. The occurrence of Rucinolithus irregularis and the absence of Eprolithus floralis or younger marker species indicates the Chiastozygus litterarius Zone of early Aptian age. Moreover, the relative dominance of nannoconids allows precise correlation to the lower $C$. litterarius Zone, which is the interval including the reversed polarity Chron M0, immediately preceding the "nannoconid crisis" (Erba, 1994) (Fig. 2).

Calcareous nannofossils apparently are absent in the interval between Cores 144-878A-74R and -42M. Two samples from Core 144878A-41M (Samples 144-878A-41M-1, 4-8 and 0-4 cm) contain frequent nannofossils with dominant Nannoconus truittii. Other taxa 
Figure 2. Age of Aptian to Albian calcareous plankton (shaded) recovered from shallow-water carbonate platform sediments at Site 878 (MIT Guyot) and at Site 879 (Takuyo-Daisan Guyot). Age scale and magnetostratigraphy after Gradstein et al (1994); nannofossil zonation of Thierstein (1973, 1976), Roth (1978), and Erba (1994); planktonic foraminifer zonation of Premoli Silva and Sliter (1994).

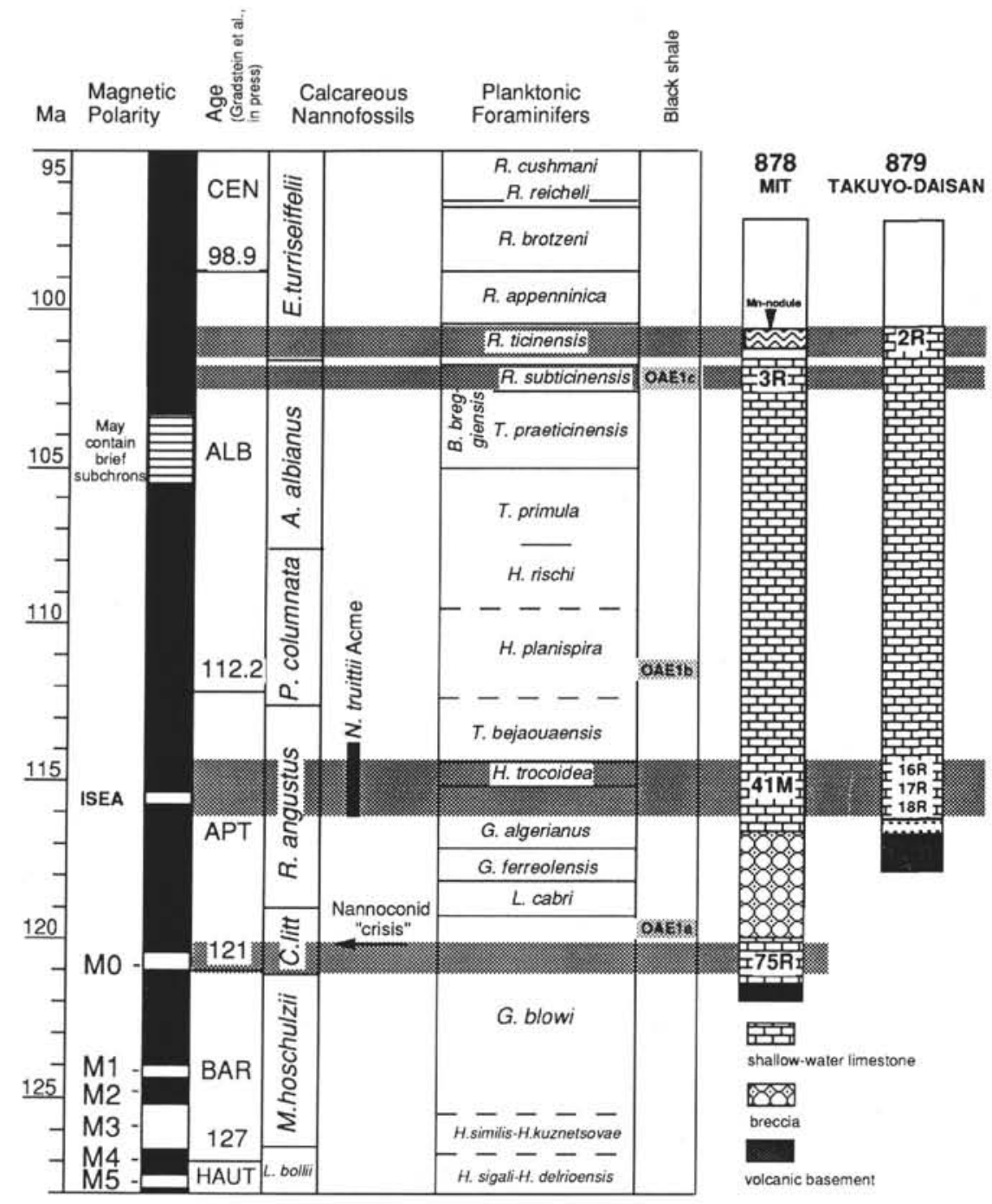

that were reported from coeval intervals in Tethyan sections (Erba, 1988), and by the presence of Braarudosphaera stenorhetha, which is restricted to the middle to late Albian (Perch-Nielsen, 1985).

\section{Planktonic Foraminifers}

A few layers belonging to both the upper and lower platform limestone yielded generally rare, poorly preserved planktonic foraminifers. They may occur more frequently than plotted in Fig. 2, but we disregarded all specimens in which the calcitic wall was not at least partially visible in thin section.

The lowermost Sample 144-878A-77R-1, 28-31 cm, yielded several specimens, mainly hedbergellids. Most are coated with a micritic, occasionally sparry, film. The species identified include common $\mathrm{Hed}$ bergella delrioensis, very rare $H$. excelsa, possible $H$. aptiana, and Globigerinelloides ferreolensis. Their average size is around $250 \mu \mathrm{m}$. A single specimen, tentatively referred to $G$. ferreolensis was observed in Samples 144-878A-75R-2, 0-4 cm, and -75R-1, 0-4 cm, respectively, whereas Sample 144-878A-75R-1, 141-143 cm, yielded more numerous planktonic foraminifers. In the latter, however, nondiagnostic sections predominate and only Hedbergella delrioensis was identified confidently.

A small planktonic foraminifer fauna occurs in Sample 144-878A$41 \mathrm{M}-1,4-8 \mathrm{~cm}$. This includes single specimens of Hedbergella similis, $H$. trocoidea, $H$. delrioensis, and Globigerinelloides barri in addition to a few unidentifiable specimens. 
Table 4. Range chart of planktonic foraminifers in the shallow-water sediments recovered at Site 873 (Wodejebato Guyot), late CampanianMaastrichtian.

\begin{tabular}{|c|c|c|c|c|c|c|c|c|c|c|c|c|c|c|}
\hline $\begin{array}{l}\text { Core, section, } \\
\text { interval }(\mathrm{cm})\end{array}$ & 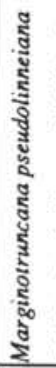 & 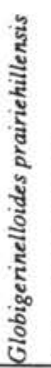 & 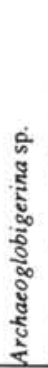 & 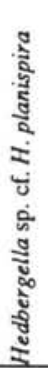 & 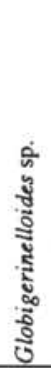 & 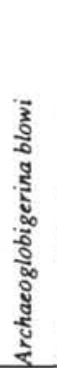 & 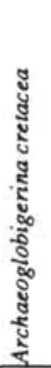 & 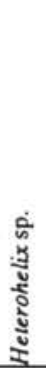 & 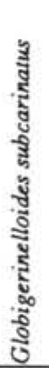 & 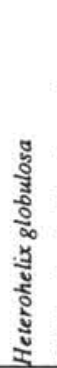 & 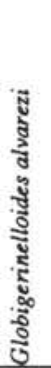 & 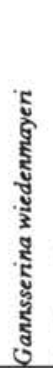 & 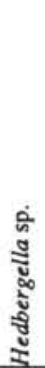 & 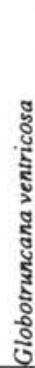 \\
\hline \multicolumn{15}{|l|}{ Hole 873B } \\
\hline $8 \mathrm{~N}-1,4-8$ & . & . & 1 & . & . & . & . & 1 & . & . & . & . & . & . \\
\hline $8 \mathrm{~N}-1,56-57$ & . & . & 1 & . & . & . & . & 1 & . & . & . & . & . & . \\
\hline $8 \mathrm{~N}-1,130-132$ & . & . & . & . & . & . & . & 2 & . & . & . & . & . & . \\
\hline $8 \mathrm{~N}-2,71-73$ & . & . & . & . & . & . & . & $?$ & . & . & . & . & . & . \\
\hline $8 \mathrm{~N}-2,77-79$ & . & . & . & . & . & . & . & 3 & . & . & . & . & 1 & . \\
\hline $8 \mathrm{~N}-\mathrm{CC}$ & . & . & . & . & . & . & . & . & . & . & . & . & . & 1 \\
\hline $9 \mathrm{~N}-1,130-135$ & & . & . & . &. & &. & 1 & . & . & . &. & 1 & \\
\hline \multicolumn{15}{|l|}{ Hole 873A } \\
\hline $3 R \cdot 1,3-6$ & . & . & . & . & . & 1 & . & . & . & . & . & . & . & . \\
\hline $4 \mathrm{R}-1,16-20$ & . & . & $?$ & . & . & . & . & . & . & . & . & . & . & . \\
\hline $5 R-1,13-18$ & . & . & 1 & . & . & . & . & . & . & . & . & $1 ?$ & . & . \\
\hline SR-1, $41-46$ & . & . & . & . & . & $?$ & . & 1 & . & . & . & . & . & . \\
\hline $5 \mathrm{R}-1,104-107$ & . & . & . & 1 & . & . & . & $?$ & . & . & . & . & . & . \\
\hline $6 \mathrm{R}-1,11-18$ & . & 1 & 1 & . & . & . & . & 1 & . & . & 1 & 1 & . & . \\
\hline $7 \mathrm{R}-1,7-9$ & . & . & 1 & . & . & . & . & 1 & . & . & . & . & . & . \\
\hline $7 R-1,16-20$ & . & . & 1 & . & . & . & . & . & . & . & . & . & . & . \\
\hline 7R-1, 30-34 & . & . & 1 & . & . & . & . & . & . & . & . & . & . & . \\
\hline $7 \mathrm{R}-2,0-4$ & . & . & 1 & . & . & . & . & . & . & . & . & . & . & . \\
\hline $8 \mathrm{R}-1,54-57$ & . & . & . & . & . & . & . & . & . & 1 & . & . & . & . \\
\hline $8 \mathrm{R}-2,0-3$ & . & . & 1 & . & . & . & . & . & . & . & . & . & . & . \\
\hline $8 R-2,20-23$ & . & . & . & . & . & . & . & 1 & . & . & . & . & . & . \\
\hline $9 \mathrm{R}-1,78-81$ & . & . & . & . & . & 1 & 1 & . & . & . & . & . & . & . \\
\hline $9 R-1,119-123$ & . & . & . & . & . & . & . & . & 1 & . & . & . & . & . \\
\hline $9 R-1,142-146$ & . & . & $\mathrm{vr}$ & . & . & . & . & . & . & . & . & . & . & . \\
\hline $9 \mathrm{R}-2,0-3$ & . & . & 1 & . & . & . & . & 2 & . & . & . & . & . & . \\
\hline $10 \mathrm{R}-1,46-50$ & 1 & . & . & . & 1 & 1 & 1 & . & . & . & . & . & . & . \\
\hline $10 \mathrm{R}-1,116-118$ & . & . & . & 1 & . & . & . & . & . & . & . & . & . & . \\
\hline $11 \mathrm{R}-2,0-3$ & 1 & 1 & 1 & & & & & & & & & & & \\
\hline
\end{tabular}

Note: Estimated abundance given as \# = number of specimens and $\mathrm{vr}=$ very rare.

Very rare, poorly preserved planktonic foraminifers were observed in Sample 144-878A-26M-1, 4-12 cm. Only two specimens could be confidently identified as Hedbergella delrioensis and Ticinella roberti, respectively.

A single specimen of Hedbergella delrioensis and a possible early form of Rotalipora subticinensis were observed in Sample 144-878A$3 \mathrm{R}-1,40-46 \mathrm{~cm}$. Sparse, small-sized planktonic foraminifers occur in Sample 144-878A-1R-CC, 13-15 cm (Fig. 2), where the species identified include single specimens of Hedbergella delrioensis, $H$. simplex, and Globigerinelloides ultramicrus.

The planktonic foraminifer assemblages at Site 878, although poorly diversified, contain a few age-diagnostic species. In particular, (1) Hedbergella excelsa and the possible H. aptiana, from the lowermost assemblage, do not range above the lower Aptian (Longoria, 1974; Banner and Desai, 1988). The mean sizes of all the individuals, not exceeding $250 \mu \mathrm{m}$, also are characteristic of lower Aptian assemblages (see Tornaghi et al., 1989). (2) The occurrence of Globigerinelloides barri and Hedbergella trocoidea dates Sample 144-878A$41 \mathrm{M}-1,4-8 \mathrm{~cm}$, and, thus the initiation of the upper carbonate plat-
Table 5. Range chart of planktonic foraminifers in the shallow-water sediments recovered at Site 874 (Wodejebato Guyot), late CampanianMaastrichtian.

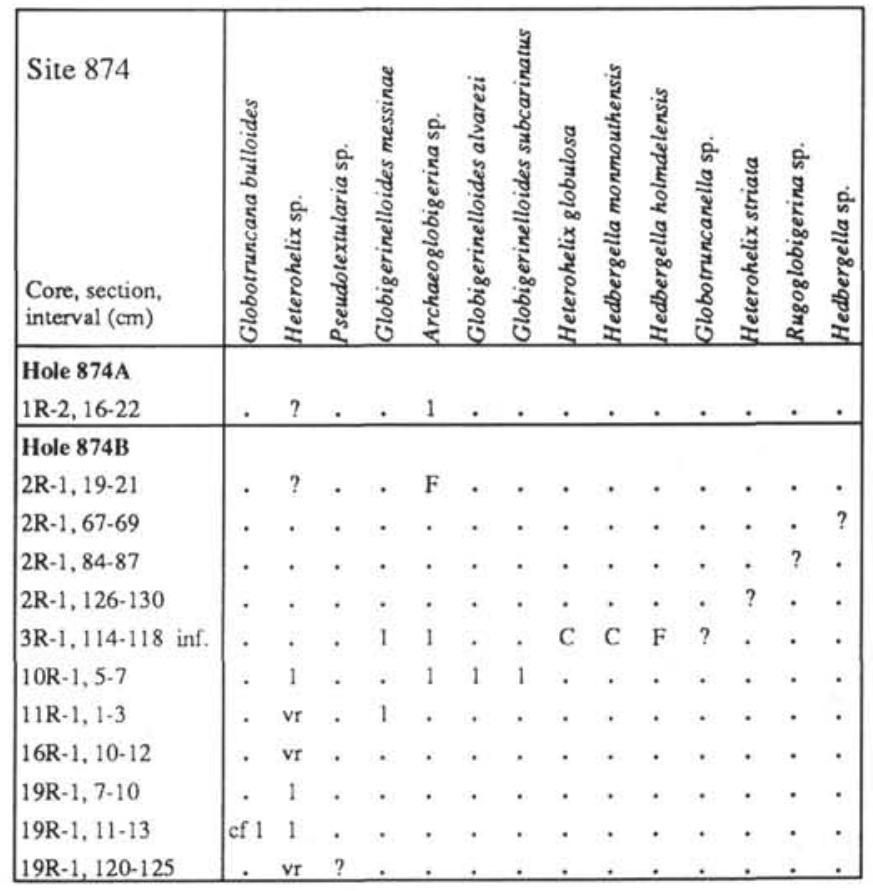

Notes: Estimated abundance given as \# = number of specimens, $v r=$ very rare, $F=f e w$, and $\mathrm{C}=$ common. "cf" = uncertain identification, and "inf." = pelagic infilling.

form, as late Aptian and possibly as middle late Aptian. This age determination is based primarily on the stratigraphic range of $G$. barri, that is known from the base of the Globigerinelloides algerianus Zone to the middle of the Hedbergella trocoidea Zone (Sigal, 1977). (3) Ticinella roberti is known to range from uppermost Aptian to upper Albian (Caron, 1985), but its distribution is not continuous, as it disappeared in the lower Albian and reappeared in the lower upper Albian Biticinella breggiensis Zone. We interpret its occurrence in Sample 144-878A-26M-1, 4-12 cm, as representing the upper part of its total range, in agreement with the Albian age inferred from the associated shallow-water benthic assemblage. (4) The tentative identification of Rotalipora subticinensis, although primitive in morphology, is diagnostic of the nominal upper subzone of the Biticinella breggiensis Zone. Sample 144-878A-3R-1, $40-46 \mathrm{~cm}$, thus, is possibly dated as late Albian.

In summary (Fig. 2), calcareous nannofossils and planktonic foraminifers indicate that (1) the initiation of carbonate platform sedimentation occurred in the earliest Aptian (lower part of the $C$. litterarius Zone before the "nannoconid crisis"), (2) the deposition of the polymictic breccia is constrained to the "middle" Aptian, (3) carbonate sedimentation resumed after the phreato-magmatic eruption, in the middle late Aptian ( N. truittii Acme, G. algerianus to H. trocoidea Zones), and (3) carbonate sedimentation ended during the late Albian after the Biticinella breggiensis Zone. This interpretation is consistent with the occurrence of Planomalina praebuxtorfi, a short-ranging species that straddles the Rotalipora ticinensis/Rotalipora appenninica zonal boundary, in the overlying manganese nodules (Fig. 2) (see Watkins et al., this volume).

\section{SITE 879 (TAKUYO-DAISAN GUYOT)}

Site 879 was drilled on the eastern margin of Takuyo-Daisan Guyot and penetrated a middle Cretaceous carbonate platform overlying the volcanic substrate. No pelagic cap was recovered at this site. Both 
Table 6. Range chart of planktonic foraminifers in the shallow-water sediments recovered at Site 875 (Wodejebato Guyot), Gansserina gansseri Zone, Maastrichtian.

\begin{tabular}{|c|c|c|c|c|c|c|c|c|c|c|c|c|c|c|c|c|c|c|c|c|c|c|c|c|c|c|c|c|c|c|}
\hline $\begin{array}{l}\text { Core, section, } \\
\text { interval }(\mathrm{cm})\end{array}$ & 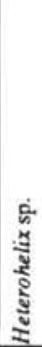 & 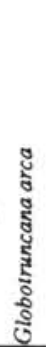 & 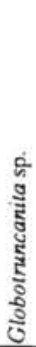 & 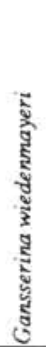 & 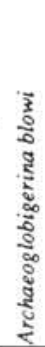 & 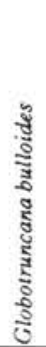 & 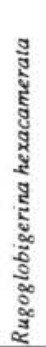 & 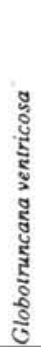 & 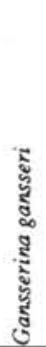 & 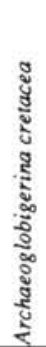 & 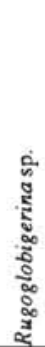 & 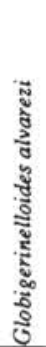 & 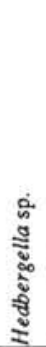 & $\begin{array}{l}\text { sू } \\
5 \\
0 \\
3 \\
0 \\
5 \\
5 \\
0 \\
0 \\
0 \\
0 \\
0 \\
0 \\
0 \\
0\end{array}$ & 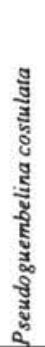 & 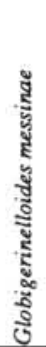 & 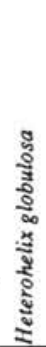 & 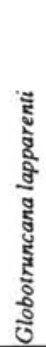 & 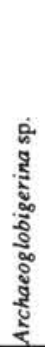 & 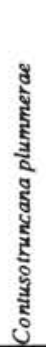 & 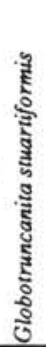 & 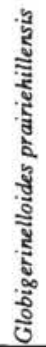 & 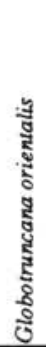 & 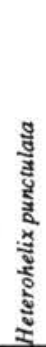 & 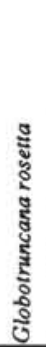 & 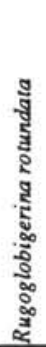 & 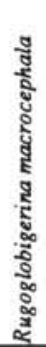 & 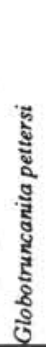 & 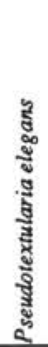 & 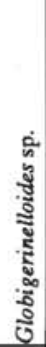 \\
\hline $1 \mathrm{M}-1,0-2$ & 1 & . & . & . & . & . & . & . & . & . & 1 & . & . & . & . & . & . & . & . & . & . & . & . & . & . & . & . & . & . & . \\
\hline $1 \mathrm{M}-1,34-36$ & 1 & . & . & . & . & . & . & . & . & . & 1 & . & . & . & . & . & . & . & 1 & . & . & . & . & . & . & . & . & . & 1 & 1 \\
\hline $2 M-1,61-64$ & 1 & . & . & . & . & . & . & . & . & . & 1 & . & . & . & . & . & . & . & 1 & . & . & . & . & . & . & . & . & . & . & . \\
\hline $4 \mathrm{M}-1,2-5$ & . & . & . & . & . & . & . & . & . & . & . & . & . & 1 & . & . & . & . & . & . & . & . & . & . & . & $\mathrm{cf}$ & . & . & . & . \\
\hline $5 \mathrm{M}-1,131-136$ & . & . & . & $?$ & . & . & . & . & . & . & . & . & . & 1 & . & 1 & 1 & . & . & . & . & . & . & 1 & . & . & . & . & . & . \\
\hline $6 \mathrm{M}-1,93-94$ & . & . & . & . & 1 & . & . & . & . & . & . & . & . & 1 & . & . & . & . & . & . & . & . & . & 1 & . & . & . & $?$ & . & . \\
\hline $7 \mathrm{M}-1,29-30$ & . & . & . & . & . & . & 1 & . & 1 & . & . & . & . & 1 & 1 & . & 1 & . & . & . & . & . & . & . & . & . & $?$ & 1 & . & . \\
\hline $7 \mathrm{M}-1,30-36$ & 1 & . & . & . & . & . & . & . & . & . & . & . & . & . & . & . & . & . & . & . & . & . & . & . & . & . & . & . & . & . \\
\hline $8 \mathrm{M}-1,18-19$ & . & . & . & . & . & , & . & . & . & . & . & . & . & . & . & . & 1 & . & . & . & . & . & . & 1 & . & . & . & . & . & . \\
\hline $9 \mathrm{M}-1,0-4$ & 1 & . & . & . & . & . & . & . & 1 & . & . & . & . & . & . & . & . & . & . & . & 1 & . & . & . & . & 1 & . & . & . & . \\
\hline $9 \mathrm{M}-1,0-7$ & . & 1 & . & . & 1 & . & . & . & . & 1 & . & . & . & 1 & . & . & . & . & . & . & . & 1 & . & . & . & . & . & . & . & . \\
\hline $9 \mathrm{M}-1,29-31$ & $\mathrm{R}$ & . & . & . & . & . & . & . & . & . & . & . & . & . & . & . & . & . & . & . & . & . & . & . & . & . & . & . & . & . \\
\hline $10 \mathrm{M}-1,122-125$ & . & . & . & . & . & . & 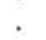 & . & . & . & . & . & . & . & 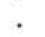 & . & . & . & . & . & . & . & . & 1 & . & . & . & . & . & . \\
\hline $10 \mathrm{M}-2,94-96$ & . & . & . & . & . & . & . & 1 & . & . & 1 & . & . & . & . & . & 1 & . & . & . & . & . & . & . & $\mathrm{cf}$ & . & . & . & . & . \\
\hline $11 \mathrm{M}-1,68-71$ & . & . & . & . & . & . & 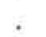 & . & . & . & . & . & . & . & . & . & . & . & 1 & . & . & . & . & . & . & . & . & . & . & . \\
\hline $11 \mathrm{M}-1,121-124$ & . & . & . & . & . & 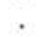 & . & . & . & . & 1 & . & . & . & . & . & . & . & 1 & . & . & . & . & 1 & . & . & . & . & . & . \\
\hline $11 \mathrm{M}-2,61-64$ & . & . & . & . & . & . & 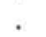 & . & . & . & . & . & . & . & . & . & $\mathrm{R}$ & . & R & . & . & . & . & . & . & . & . & . & . & . \\
\hline $11 \mathrm{M}-3,49-54$ & . & . & . & . & 1 & $\cdot$ & . & 1 & . & . & . & . & . & . & . & . & 1 & . & . & . & . & . & . & 1 & . & . & . & . & . & . \\
\hline $11 \mathrm{M}-3,93-97$ & . & vr & . & . & 1 & . & . & . & 1 & 1 & . & 1 & . & . & . & . & C & . & . & . & . & 1 & 1 & . & . & . & . & . & . & . \\
\hline $12 \mathrm{M}-1,30-34$ & $\mathrm{~F}$ & . & . & . & ef & . & . & . & . & . & . & . & . & 1 & . & . & . & . & . & . & . & . & . & . & . & . & . & . & . & . \\
\hline $12 \mathrm{M}-1,131-135$ & 1 & . & . & . & . & . & . & . & . & 1 & . & . & . & . & . & . & . & . & . & . & . & . & . & . & . & . & . & . & . & . \\
\hline $12 \mathrm{M}-1$ (cuttings) & . & cf & . & . & $\mathrm{R}$ & 1 & . & . & . & . & . & . & . & . & $\cdot$ & . & $\cdot$ & . & . & 1 & cf & . & . & . & . & . & . & . & . & . \\
\hline $13 \mathrm{M}-1,24 \cdot 26$ & . & . & . & . & . & . & . & . & . & . & . & . & . & . & 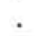 & . & . & . & 1 & . & . & . & . & . & . & . & - & . & . & . \\
\hline $13 \mathrm{M}-1,67-72$ & . & 1 & $\mathrm{~F}$ & . & . & 1 & . & $\mathrm{F}$ & $\mathrm{F}$ & 1 & . & $?$ & 1 & 1 & $\mathrm{~F}$ & 1 & 1 & $\mathrm{~F}$ & . & . & . & . & . & . & . & . & . & . & . & . \\
\hline $13 \mathrm{M}-1,68-73$ & . & . & . & $\mathrm{F}$ & $\mathrm{C}$ & C & . & . & . & C & $\mathrm{C}$ & $\mathrm{F}$ & C & . & . & , & . & . & . & . & . & . & . & . & . & . & . & . & . & . \\
\hline $13 \mathrm{M}-1,123-130$ & . & . & . & . & 1 & 1 & . & . & . & . & . & . & . & . & . & . & . & . & . & . & . & . & . & . & . & . & . & . & . & . \\
\hline $13 \mathrm{M}-1,125-129$ & . & 2 & . & . & . & 2 & $\mathrm{~F}$ & $\mathrm{~F}$ & $\mathrm{~F}$ & . & . & . & . & . & . & . & . & . & . & . & . & . & . & . & . & . & . & . & . & . \\
\hline $13 \mathrm{M}-2,61-64$ & . & . & $\mathrm{R}$ & $\mathrm{R}$ & 1 & . & . & . & . & . & . & . & . & . & . & . & . & . & . & . & . & . & . & . & . & . & . & . & . & . \\
\hline $14 \mathrm{M}-1,15-17$ & 1 & . & . & . & . & . & . & . & . & . & , & . & . & . & . & . & . & . & . & . & . & . & . & . & . & . & . & . & . & - \\
\hline $14 \mathrm{M}-1,50-53$ & ? & ? & . & . & . & . & . & . & . & . & . & . & . & . & . & . & . & . & . & . & . & . & . & . & . & . & . & . & - & . \\
\hline $14 \mathrm{M}-1,59-62$ & $?$ & . & . & . & . & . & . & . & . & . & . & . & . & . & . & . & . & . & . & & & & . & . & . & . & . & . & . & . \\
\hline
\end{tabular}

Notes: Estimated abundance given as \# = number of specimens, $\mathrm{vr}=$ very rare, $\mathrm{R}=\mathrm{rare}, \mathrm{F}=\mathrm{few}$, and $\mathrm{C}=$ common. "cf" $=$ uncertain identification.

calcareous nannofloras and planktonic foraminifers were encountered in the oldest sediments overlying basalts and provide a precise biostratigraphic age for the inception of marine sedimentation.

\section{Calcareous Nannofossils}

At Site 879 , calcareous nannofossils were extensively sought in smear slides and thin sections from the upper and lower parts of the shallow-water sequence (Table 10). A few nannofossiliferous layers occur in Cores 144-879A-16R, -17R, and -18R, from the oldest sediments above the volcanic substrate. The best nannofossil assemblages are in Samples 144-879A-17R-1, 70-80, 73-74, 80-83, and 97-103 $\mathrm{cm}$, which contain Eprolithus floralis, Watznaueria barnesae, Watznaueria britannica, Micrantolithus hoschulzii, Zygodiscus erectus, Vagalapilla stradneri, Biscutum constans, Diazomatholithus lehmanii, Microstaurus chiastius, Lithraphidites carniolensis, Discorhabdus rotatorius, Braarudosphaera africana, Nannoconus truittii, Rucinolithus irregularis, Rucinolithus terebrodentarius, Rhagodiscus asper, Rhagodiscus angustus, and Markalius circumradiatus. The occurrence of Eprolithus floralis without younger marker species indicates the upper Aptian Rhagodiscus angustus Zone. The relative dominance of Nannoconus truittii in all these sample indicate that Core 144-879A-17R is correlative with the middle late Aptian $N$. truittii
Acme. Similar, although depauperate, nannofloras occur in Samples 144-879A-18R-1, 30-35 and $31-37 \mathrm{~cm}$; these are consequently attributed to the $N$. truittii Acme. Most samples from Core 144-879A16R lack calcareous nannofossils. Sparse specimens of Nannoconus truittii, Watznaueria barnesae, Rucinolithus irregularis, and Eprolithus floralis were recorded in Samples 144-879A-16R-1, 25-26, $27-33$, and $68-72 \mathrm{~cm}$. Again, although a precise age assignment is biased by the paucity of nannofossils, the relative dominance of Nannoconus truittii suggests that the middle upper Aptian N. truittii Acme may extend to Core 144-879A-16R (Fig. 2).

Calcareous nannofossils were not observed in any samples investigated from the upper shallow-water limestone at Site 879.

\section{Planktonic Foraminifers}

Planktonic foraminifers occur in several layers in the lowermost cores and close to the top of the platform sequence. They are very rare to rare, except in two samples from Sections 144-879A-18R-1 and $-16 \mathrm{R}-1$, where modest numbers occur. Preservation is poor overall.

The lowermost Sample 144-879A-18R-1, 22-26 cm, yielded few specimens mainly attributed to small, indeterminate hedbergellid cross sections. Two specimens are attributed to Hedbergella planispira and the $H$. trocoidea group, respectively. 
Table 7. Range chart of planktonic foraminifers in the shallow-water sediments recovered at Site 876 (Wodejebato Guyot), Gansserina gansseri Zone, Maastrichtian.

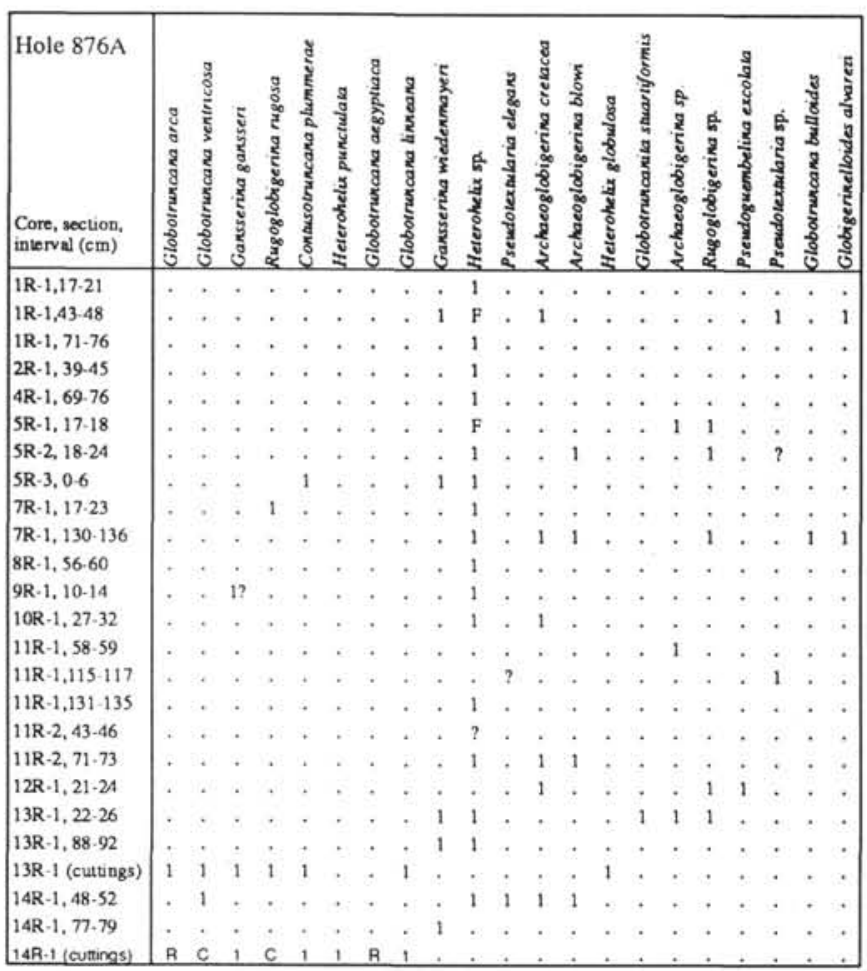

Notes: Estimated abundance given as \# = number of specimens, $\mathrm{R}=\mathrm{rare}, \mathrm{F}=\mathrm{few}$, and $\mathrm{C}$ $=$ common.

A possible Globigerinelloides algerianus and Hedbergella delrioensis occur in Sample 144-879A-18R-1, 10-13 cm, along with other rare unidentifiable forms. Single, dubious specimens of Globigerinelloides, Hedbergella, and lagenids were observed in Sample 144$879 \mathrm{~A}-18 \mathrm{R}-1,4-7 \mathrm{~cm}$. Very rare indeterminate planktonic foraminifers also occur in Samples 144-879A-17R-1, 109-112 and 0-5 cm.

One of the very rare specimens observed in Sample 144-879A$16 \mathrm{R}-1,123-125 \mathrm{~cm}$, possesses large kidney-shaped chambers in crosssection and may be attributable either to Globigerinelloides barri or to H. trocoidea. The latter species, larger than $300 \mu \mathrm{m}$ in size, also was identified in Sample 144-879A-16R-1, 79-84 cm, along with a Globigerinelloides, possibly $G$. ferreolensis. In the richest assemblage from Sample 144-879A-16R-1, 27-33 cm, on the contrary, nondiagnostic cross-sections prevented identification of any specimen at the specific level; most were attributed to hedbergellids.

Finally, a large specimen $(>500 \mu \mathrm{m})$ with an acute, possibly keeled, margin, a long, slightly convex spiral side, chambers that gradually increase in size, and a large umbilicus was recorded in Sample 144$879 \mathrm{~A}-2 \mathrm{R}-1,132-138 \mathrm{~cm}$, is tentatively referred to Rotalipora ticinensis. A second specimen somewhat resembles Ticinella raynaudi, and a third belongs to the genus Hedbergella.

Planktonic foraminifer assemblages from the lower cores (144879A-18R to -16R), although at low abundance and diversity, appear homogeneous. The occurrence of forms attributed to $H$. trocoidea, $G$. ferreolensis, and possibly to G. algerianus and G. barri would indicate the Globigerinelloides algerianus Zone and constrain the age of the lowermost interval to the middle late Aptian (Figure 2). The presence of $R$. ticinensis in Sample 144-879A-2R-1, 132-138 cm, if correct, would indicate the late Albian Rotalipora ticinensis Zone (Fig. 2).

In summary, calcareous nannofossils and planktonic foraminifers suggest that the initiation of carbonate platform sedimentation at Site 879 occurred during the middle late Aptian (N. truittii Acme, $G$.
Table 8. Range chart of planktonic foraminifers in the shallow-water sediments recovered at Site 877 (Wodejebato Guyot), late CampanianMaastrichtian.

\begin{tabular}{|c|c|c|c|c|c|c|c|c|c|c|c|c|c|c|c|c|}
\hline $\begin{array}{l}\text { Core, section, } \\
\text { interval }(\mathrm{cm})\end{array}$ & 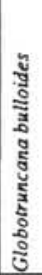 & 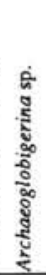 & 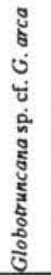 & 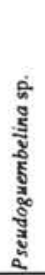 & 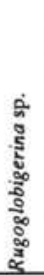 & 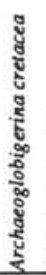 & 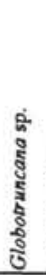 & 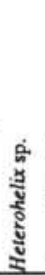 & 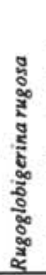 & 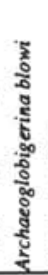 & 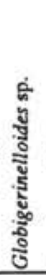 & 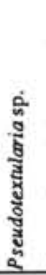 & 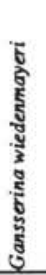 & 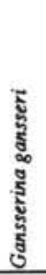 & 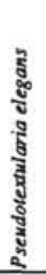 & 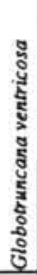 \\
\hline $1 R-1,8-10$ & . & . & . & . & . & . & . & . & . & . & . & . & . & cf & 1 & 1 \\
\hline $1 R-2,42 \cdot 46$ & 1 & 1 & . & . & . & . & . & . & . & . & . & . & $?$ & . & . & . \\
\hline $2 R \cdot 1,53 \cdot 55$ & . & 1 & . & . & . & . & . & $?$ & . & . & . & 1 & . & . & . & . \\
\hline $3 R-1,130-133$ & . & . & . & . & $?$ & . & . & & . & . & . & 1 & . & . & . & . \\
\hline $4 R \cdot 2,18 \cdot 21$ & . & . & . & . & . & . & . & 1 & . & . & 1 & . & . & . & . & . \\
\hline 4R-3, 13-17 & . & . & . & . & . & . & . & 1 & . & . & 1 & . & . & . & . & . \\
\hline SR-1, 8-4 & . & . & . & . & . & . & . & $?$ & . & . & . & . & . & . & . & . \\
\hline 7R-1,88-91 & . & . & . & . & . & . & . & 1 & . & . & 1 & . & . & . & . & . \\
\hline $7 R-2,109-120$ & . & . & $?$ & . & . & . & . & . & 1 & 1 & . & . & . & . & . & . \\
\hline 10R-1, 3-10 & . & . & . & . & . & $\mathbf{R}$ & . & . & . & . & . & . & . & . & . & . \\
\hline $18 \mathrm{R}-1,25-28$ & . & 1 & . & . & . & c & 1 & 1 & . & . & . & . & . & . & . & . \\
\hline $18 R \cdot 1,70.74$ & 1 & 1 & . & . & $?$ & 1 & - & . & . & . & . & . & . & . & . & . \\
\hline $19 \mathrm{R}-1,27-30$ & . & $?$ & . & $?$ & $?$ & . & . & . & . & . & . & . & . & . & . & . \\
\hline $20 \mathrm{R}-1,0-7$ & . & . & 1 & . & . & . & . & . & . & . & . & . & . & . & . & . \\
\hline $20 R-1,61.63$ & $\mathrm{vr}$ & 1 & &. & & . & & . & & . & & & . & & . & \\
\hline
\end{tabular}

Notes: Estimated abundance given as \# = number of specimens, $\mathrm{vr}=$ very rare, $\mathrm{R}=$ rare, and $\mathrm{C}=$ common. "cf" = uncertain identification.

algerianus to $H$. trocoidea Zones) and ended by late Albian ( $R$. ticinensis Zone). The shallow-water limestone at Site 879 is correlative with the upper platform sequence recovered in Hole 878A. The poor recovery in the middle part of the cored sequence at Site 879 prevents the identification of possible hiatuses. In fact, the carbonate sequence at this site is only half the thickness of that deposited at Site 878 on MIT Guyot during the late Aptian to late Albian interval.

\section{CRETACEOUS CALCAREOUS PLANKTON BIOSTRATIGRAPHY AND CHRONOSTRATIGRAPHY}

Cretaceous stratigraphy currently is being revised to improve the correlation between chronostratigraphy and biomagnetostratigraphy, using data from sections in Tunisia, Spain, northern Germany, and North America. These sections provide ammonite control for calcareous plankton biostratigraphy and/or magnetostratigraphy. This revision has also recalibrated the Late Cretaceous stage boundaries with respect to magnetic polarity chronozones and/or calcareous plankton biozones (Premoli Silva and Sliter, 1994). Moreover, a revised time scale has been proposed (Gradstein et al., 1994) with updated absolute ages for Mesozoic stage boundaries. Figure 1 illustrates the revised geochronology and biomagnetostratigraphy of the Late Cretaceous and the stratigraphic position of the calcareous planktonbearing intervals at Sites 872 to 877 .

We continue to equate the base of the Rotalipora brotzeni Zone with the Albian/Cenomanian boundary (e.g., Sigal, 1977; Porthault, 1978 ) as there is little macropaleontologic data and no nannofossil events at this boundary. Further, both stages fall in the Cretaceous Normal Polarity Superchron. The Cenomanian/Turonian boundary falls within the Whiteinella archaeocretacea Zone (Elder and Kirkland, 1985; Leckie, 1985; Robazynski, 1989); the first occurrence of the calcarerous nannofossil Quadrum gartneri shortly precedes this boundary. The Helvetoglobotruncana helvetica Zone begins in the lowermost Turonian and extends to the upper half of the middle Turonian (Robazynski et al., 1990). The Marginotruncana sigali Zone is con- 
Table 9. Range chart of calcareous nannofossils in the shallow-water limestones recovered at Site 878 (MIT Guyot).

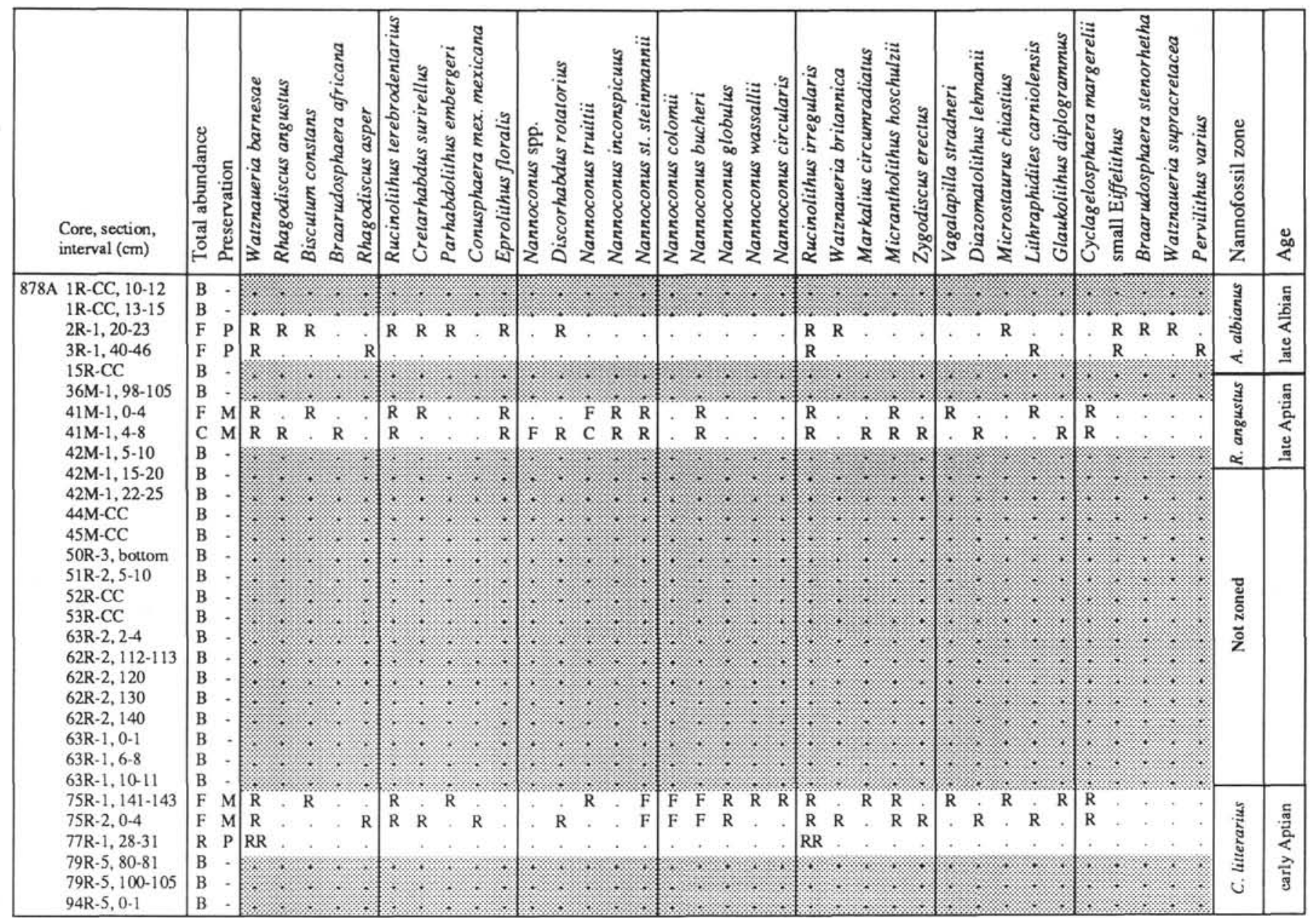

Notes: Shaded areas are barren of nannofossils. Estimates of the total nannofossil abundance are given as $\mathrm{C}(\mathrm{common})=1 \%-10 \%$ of all particles, $\mathrm{F}$ (few) $=0.1 \%-1 \%$ of all particles, and $\mathrm{R}$ (rare) $=<0.1 \%$ of all particles. Estimates of preservation are given as $\mathrm{M}$ (moderate) $=$ most specimens display moderate overgrowth/dissolution, and species identification is usually not impaired; and $\mathrm{P}$ (poor) = most specimens display significant amounts of overgrowth/dissolution, and species identification is sometimes impaired. Estimates of the relative abundance of nannofossil species are given as $C$ (common) $=1-10$ specimens per field of view, $F($ few $)=$ one specimen per $2-10$ fields of view, $R$ (rare) $=$ one specimen per 11-100 fields of view, and RR (extremely rare) $=$ one specimen per $>100$ fields of view.

fined to the middle Turonian (Robazynski et al., 1990). The base of the Dicarinella concavata Zone correlates to the base of the upper Turonian, and the zone extends from the upper Turonian through the Coniacian, possibly straddling the Coniacian/Santonian boundary (Robazynski et al., 1990; Salaj, 1980, reinterpreted). The base of the Dicarinella asymetrica (= carinata of Salaj, 1980) Zone falls very close to the base of the Santonian (Salaj, 1980, 1984); this position is supported by the first occurrence of Sigalia and ventilabrellids slightly above the base of the zone in the Tunisian sections; the latter event in the Bottaccione section of central Italy, is almost coincident with the first occurrence of D. asymetrica (Premoli Silva and Sliter, 1994). No nannofossil event coincides with the Coniacian/Santonian boundary, which falls between the first occurrence of Micula decussata (late Coniacian) and the first occurrence of Lithastrinus grillii and Reinhardtites anthophorus (earliest Santonian). The calcareous nannofossil Aspidolithus parcus parcus appears almost at the top of the Cretaceous Normal Polarity Superchron in the Bottaccione section (Ghisletti and Erba, unpubl. data) in agreement with the record in northern Germany (Svabenicka, 1991) and approximates the base of the Campanian. The top of the Dicarinella asymetrica Zone falls within the lower Campanian (Lilligraven, 1991; Lommerzheim and Hambach, 1992) and in the Bottaccione section equates to the termination of the Cretaceous Normal Polarity Superchron. The Campanian/
Maastrichtian boundary equates to the magnetic Chron 32N/Chron 31R boundary (Lommerzheim and Hambach, 1992; Gradstein et al., 1994), which falls within the Gansserina gansseri Zone in the Bottaccione section; this is in agreement with ammonite, calcareous nannofossil, planktonic foraminifer, and paleomagnetic correlations in Spain (Mary et al., 1991; Burnett et al., 1992a, 1992b). Following this correlation, Premoli Silva and Sliter (1994) suggested that the Campanian/ Maastrichtian boundary in the Bottaccione sequence of central Italy is best approximated by the FO of the planoglobulinids. The last occurrence of Globigerinelloides bollii correlates with the late Campanian and the first occurrence of Heterohelix navarroensis and Contusotruncana contusa above it. The last occurrences of the nannofossil taxa Tranolithus phacelosus and Quadrum trifidum occur in reversed polarity Subchron $32 \mathrm{R} 1 \mathrm{n}$ and seem to be the best events to approximate the Campanian/Maastrichtian boundary.

These new correlations imply that the Radotruncana calcarata Zone no longer represents the uppermost zone of the Campanian as previously considered and, moreover, that the Globotruncanella havanensis and Globotruncana aegyptiaca Zones and the lower half of the Gansserina gansseri Zone also are late Campanian in age (Lommerzheim and Hambach, 1992). This placement is in agreement with correlations recorded in North America (Hicks et al., 1991; Obradovich, 1991; Gradstein et al., 1994). 
Table 10. Range chart of calcareous nannofossils in the shallow-water limestones recovered at Site 879 (Takuyo-Daisan Guyot).

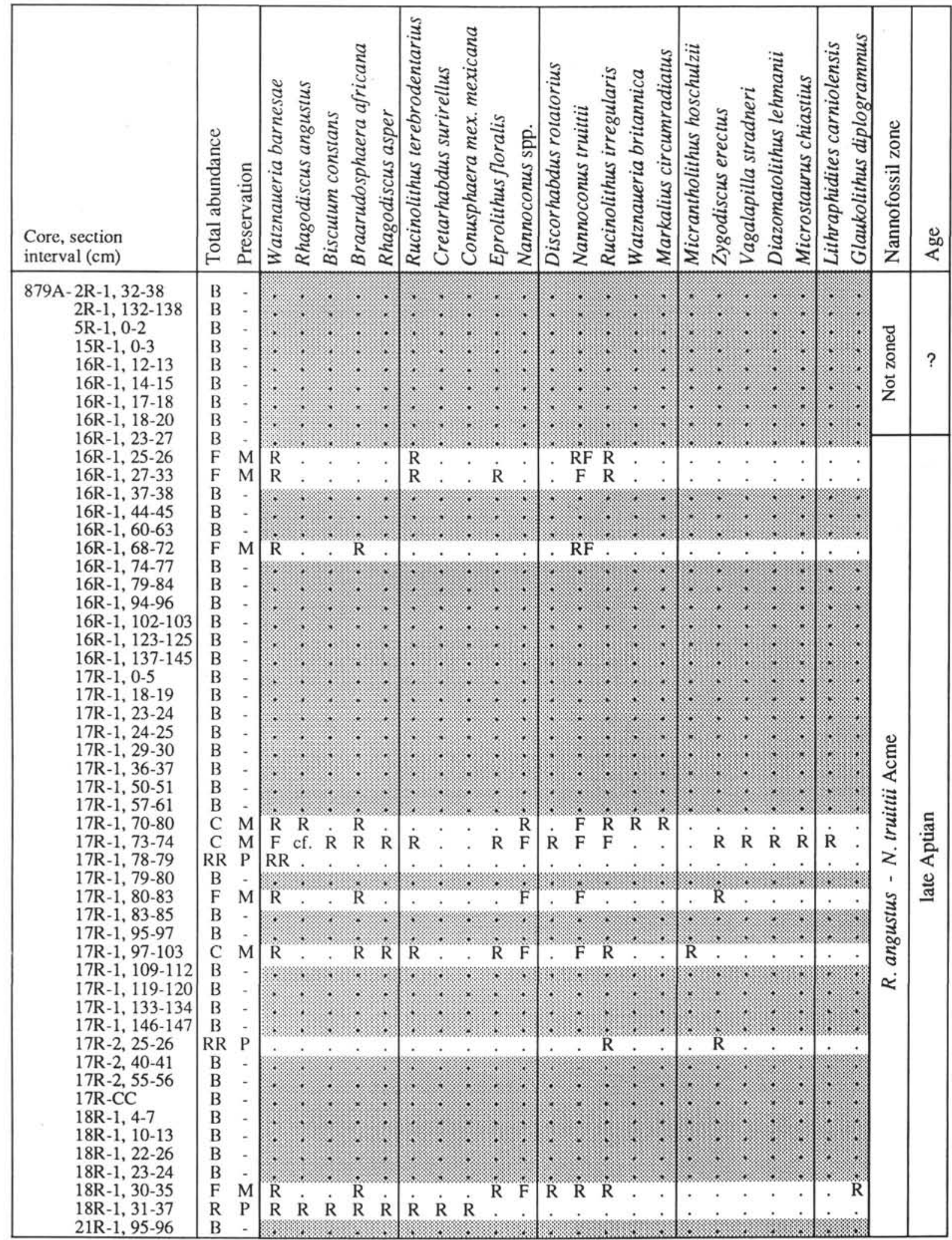

Note: Shaded areas are barren of nannofossils. Estimates of the total nannofossil abundance are given as $\mathrm{C}(\mathrm{common})=1 \%-10 \%$ of all particles, $\mathrm{F}$ (few) $=0.1 \%-1 \%$ of all particles, $\mathrm{R}$ (rare) $=<0.1 \%$ of all particles, and $\mathrm{RR}$ (extremely rare) $=<0.01 \%$ of all particles. Estimates of preservation are given as $\mathrm{M}$ (moderate) $=$ most specimens display moderate overgrowth/dissolution, and species identification is usually not impaired; and $\mathrm{P}$ (poor) = most specimens display significant amounts of overgrowth/dissolution, and species identification is sometimes impaired. Estimates of the relative abundance of nannofossil species are given as $\mathrm{F}(\mathrm{few})=$ one specimen per $2-10$ fields of view, $\mathrm{R}$ (rare) $=$ one specimen per $11-100$ fields of view, and $\mathrm{RR}(\mathrm{extremely}$ rare) $=$ one specimen per $>100$ fields of view. 
For sites drilled during Leg 144, some of the preliminary age assignments in Premoli Silva, Haggerty, Rack, et al. (1993) based on calcareous plankton from Upper Cretaceous sediments must be revised.

At Site 872 (Lo-En Guyot) the pelagic limestone infilling fractures in the basaltic substrate are attributed to the planktonic foraminifer $D$. asymetrica and $D$. concavata zones and to the nannofossil Zones $\mathrm{CC} 14-\mathrm{CC} 17$. The oldest biostratigraphic age of these sediments is late Coniacian to earliest Santonian (D. concavata planktonic foraminifer zone; $\mathrm{CC} 14-\mathrm{CC} 15$ nannofossil Zones).

The oldest marine sediments on Wodejebato Guyot originally were dated as latest Campanian, but now are attributed to the middle late Campanian (Fig. 1). In fact, the nannofossil Zone CC22 of Sissingh (1977) recognized in Core 144-877A-20R is now revised to a middle late Campanian age.

\section{CONCLUSIONS}

Detailed investigation of calcareous plankton in samples from Cretaceous carbonate platform sequences at Sites 872, 873, 874, $875,876,877,878$, and 879 resulted in important biostratigraphic age constraints for the onset, development, and demise of shallow-water sedimentation on Cretaceous guyots in the central and western Pacific Ocean.

On Wodejebato Guyot planktonic foraminifers are scattered throughout the shallow-water sequences at Sites 873 through 877 . However, calcareous nannofossils were observed only at Sites 873 , 874 , and 877 , where they occur exclusively in the oldest marine sediments immediately overlying the volcanic substratum. Calcareous plankton "iostratigraphy indicates that the initiation of carbonate platform sedimentation occurred in the late Campanian (CC22 Zone of Sissingh, 1977), continued during the late Campanian and Maastrichtian, and ended during the latest Maastrichtian.

On MIT Guyot (Site 878) calcareous nannofossils and planktonic foraminifers are present at various levels in the shallow-water limestone sequence. They indicate that the initiation of carbonate platform sedimentation occurred in the earliest Aptian (lower part of the $C$. litterarius Zone before the "nannoconid crisis"). After the phreatomagmatic eruption that deposited a polymictic breccia, carbonate sedimentation resumed in the middle late Aptian ( $N$. truittii Acme, $G$. algerianus to $H$. trocoidea Zones). Shallow-water deposition ended during the late Albian after the Biticinella breggiensis Zone. This interpretation is consistent with the occurrence of Planomalina praebuxtorfi, a species with a very short range that straddles the Rotalipora ticinensis/Rotalipora appenninica zonal boundary, in the overlying manganese nodules (see Watkins et al., this volume).

Based on calcareous plankton biostratigraphy, the initiation of carbonate platform sedimentation at Site 879 occurred during the middle late Aptian ( $N$. truittii Acme, G. algerianus to H. trocoidea Zones) and ended by the late Albian ( $R$. ticinensis Zone). The shallow-water limestone at Site 879 correlates with the upper platform sequence recovered in Hole $878 \mathrm{~A}$. The thickness of the carbonate sequence at this site is only half of that deposited at Site 878 on MIT Guyot during the late Aptian to late Albian interval suggesting possible hiatuses.

Lo-En (Site 872) is the only guyot where shallow-water limestone was not recovered during Leg 144 . Here, the oldest sediments consist of pelagic limestones infilling fractures within the volcanic substrate. Calcareous plankton biostratigraphy constrains the age of these sediments to late Coniacian to earliest Santonian (D. concavata planktonic foraminifer zone; CC14-CC15 nannofossil zones).

\section{ACKNOWLEDGMENTS}

The Ocean Drilling Program is greatly acknowledged for inviting us to participate on Leg 144. We are grateful to Brian Huber, Bill Sliter, and Percy Strong for the careful review of the manuscript. Research was supported by CNR funds to EE and IPS.

\section{REFERENCES}

Banner, F.T., and Desai, D., 1988. A review and revision of the Jurassic-Early Cretaceous Globigerina, with especial reference to the Aptian assemblages of Speeton (North Yorkshire, England). J. Micropaleontol., 7:143-185.

Bralower, T.J., 1993. Aptian-Albian calcareous nannofossil biostratigraphy of ODP Site 763 and the correlation between high- and low-latitude zonations. In Duncan, R.A., Rea, D.K., Kidd, R.B., von Rad, U., and Weissel, J.K. (Eds.), Synthesis of Results from Scientific Drilling in the Indian Ocean. Geophys. Monogr., Am. Geophys. Union, 70:245-252.

Bralower, T.J., Sliter, W.V., Arthur, M.A., Leckie, R.M., Allard, D.J., and Schlanger, S.O., 1993. Dysoxic/anoxic episodes in the Aptian-Albian (Early Cretaceous). In Pringle, M.S., Sager, W.W., Sliter, M.V., and Stein, S. (Eds.), The Mesozoic Pacific: Geology, Tectonics, and Volcanism. Geophys. Monogr., Am. Geophys. Union, 77:5-37.

Burnett, J.A., Hancock, J.M., Kennedy, W.J., and Lord, A.R., 1992a. Macrofossil, planktonic foraminiferal and nannofossil zonation at the Campanian/Maastrichtian boundary. Newsl. Stratigr., 27:157-172.

Burnett, J.A., Kennedy, W.J., and Ward, P.D., 1992b. Maastrichtian nannofossil biostratigraphy in the Biscay region (southwestern France, northern Spain). Newsl. Stratigr., 26:145-155.

Caron, M., 1985. Cretaceous planktic foraminifers. In Bolli, H.M., Saunders, J.B., and Perch-Nielsen, K. (Eds.), Plankton Stratigraphy: Cambridge (Cambridge Univ. Press), 17-86.

Caron, M., and Homewood, P., 1983. Evolution of early planktic foraminifers. Mar. Micropaleontol., 7:453-462.

Coccioni, R., Erba, E., and Premoli Silva, I., 1992. Barremian-Aptian calcareous plankton biostratigraphy from the Gorgo Cerbara section (Marche, central Italy) and implications for plankton evolution. Cretaceous Res., 13:517-537.

Elder, W.P., and Kirkland, J.I., 1985. Stratigraphy and depositional environments of the Bridge Creek Limestone Member of the Greenhorn Limestone at Rock Canyon Anticline near Pueblo, Colorado. In Pratt, L., Kauffman, E., and Zelt, F. (Eds.), Fine-grained Deposits of the Cretaceous Western Interior Seaway: Evidence of Cyclic Sedimentary Processes. Soc. Econ. Paleontol. Mineral., Field Trip Guidebook, 4:122-134.

Erba, E., 1988. Aptian-Albian calcareous nannofossil biostratigraphy of the Scisti a Fucoidi cored at Piobbico (Central Italy). Riv. Ital. Paleontol. Stratigr., 94:249-284.

Erba, E., 1994. Nannofossils and superplumes: the early Aptian "nannoconid crisis." Paleoceanography, 9:483-501.

Gradstein, F.M., Agterberg, F.P., Ogg, J.G., Hardenbol, J., and Huang, Z., 1994. A Mesozoic time scale. J. Geophys. Res., 99:24051-24074.

Hicks, J.F., Tauxe L., and Obradovich, J.D., 1991. The magnetostratigraphy of the Pierre Shale (Campanian-Maastrichtian) at the Red Bird section in eastern Wyoming. Geol. Soc. Am. Abstr., A420.

Leckie, R.M., 1985. Foraminifera of the Cenomanian/Turonian boundary interval, Greenhorn Formation, Rock Canyon anticline, Pueblo, Colorado. In Pratt, L.M., Kauffman, E.G., and Zelt, F.B. (Eds.), Fine-grained Deposits and Biofacies of the Cretaceous Western Interior Seaway: Evidence of Cyclic Sedimentary Processes. Soc. Econ. Paleontol. Mineral., Field Trip Guidebook, 4:139-150.

Lillegraven, J.A., 1991. Stratigraphic placement of the Santonian/Campanian boundary (Upper Cretaceous) in the North American Gulf Coastal Plain and Western Interior, with implications to global geochronology. Cretaceous Res., 12:115-136.

Lincoln, J.M., Pringle, M.S., and Premoli-Silva, I., 1993. Early and Late Cretaceous volcanism and reef-building in the Marshall Islands. In Pringle, M.S., Sager, W.W., Sliter, W.V., and Stein, S. (Eds.), The Mesozoic Pacific: Geology, Tectonics, and Volcanism. Geophys. Monogr., Am. Geophys. Union, 77:279-305.

Lommerzheim, A.J., and Hambach, U., 1992. Cephalopod- and magnetostratigraphy of the Campanian of the Muensterland Basin, NW-Germany. Cretaceous Symp., Hamburg 28 Sept.-3 Oct. 1991. Abstr. Vol.

Longoria, J.F., 1974. Stratigraphic, morphologic and taxonomic studies of Aptian planktonic Foraminifera. Rev. Esp. Micropaleontol., Num. Extraordinario.

\footnotetext{
- Abbreviations for names of organizations and publications in ODP reference lists follow the style given in Chemical Abstracts Service Source Index (published by American Chemical Society).
} 
Mary, C., Moreau, M.-G., Orue-Extabarria, X., Apellaniz, E., and Courtillot, V., 1991. Biostratigraphy and magnetostratigraphy of the Cretaceous/ Tertiary Sopelana section (Basque country). Earth Planet. Sci. Lett., 106:133-150.

Mutterlose, J., 1989. Temperature-controlled migration of calcareous nannofossils in the northwest European Aptian. In Crux, J.A., and van Heck, S.E. (Eds.), Nannofossils and Their Applications: Chichester (Ellis Horwood), $122-142$.

, 1991. Das Verteilungs- und Migrationsmuster des kalkigen Nannoplanktons in der borealen Unterkreide (Valangin.-Apt.) NW-Deutschlands. Palaeontographica B, 221:27-152.

Nakanishi, M., Gee, J., and ODP Leg 144 Shipboard Scientific Party, 1992. Paleolatitude of guyots in the northwestern Pacific Ocean: preliminary results of ODP Leg 144. Eos, 73:586-587.

Obradovich, J.D., 1991. A revised Cenomanian-Turonian time scale based on studies from the Western Interior United States. Geol. Soc. Am. Abstr Progr., A296.

ODP Leg 144 Shipboard Scientific Party, 1993. Insight on the formation of Pacific guyots from ODP Leg 144. Eos, 74:358-366.

Perch-Nielsen, K., 1985. Mesozoic calcareous nannofossils. In Bolli, H.M., Saunders, J.B., and Perch-Nielsen, K. (Eds.), Plankton Stratigraphy: Cambridge (Cambridge Univ. Press), 329-426.

Porthault, B., 1978. Foraminiferes caractéristiques du Cénomanien à daciès pélagique dans le Sud-Est de la France. Geol. Mediterr., 5:183-194.

Premoli Silva, I., Haggerty, J., Rack, F., et al., 1993. Proc. ODP, Init. Repts., 144: College Station, TX (Ocean Drilling Program).

Premoli Silva, I., and Sliter, W.V., 1994. Cretaceous planktonic foraminiferal biostratigraphy and evolutionary trends from the Bottaccione Section, Gubbio, Italy. Palaeontogr. Ital., 81:2-90.

Robaszynski, F., 1989. Evénements à l' échelle globale pendant la partie moyenne du Crétacé. Geobios, Mem. Spec., 11:311-319.

Robaszynski, F., Caron, M., Dupuis, C., Amedro, F., Gonzales-Donoso, J.M. Linares, D., Hardenbol, J., Gartner, S., Calandra, F., and Deloffre, R., 1990.
A tentative integrated stratigraphy in the Turonian of central Tunisia: formations, zones and sequential stratigraphy in the Kalaat Senan area. Bull. Cent. Rech. Expl.-Prod. Elf-Aquitaine, 14:213-384.

Roth, P.H., 1978. Cretaceous nannoplankton biostratigraphy and oceanography of the northwestern Atlantic Ocean. In Benson, W.E., Sheridan, R.D., et al., Init. Repts. DSDP, 44: Washington (U.S. Govt. Printing Office), 279-305.

Salaj, J., 1980. Microbiostratigraphie du Crétacé et du Paléogène de la Tunisie septentrionale et orientale (Hypostratotypes tunisiens). Geol. Ustav Dionyza Stura.

1984. Boundaries of Upper Cretaceous hypostratotypes at the profile Djebel Fguria Salah, Tunisia. Bull. Geol. Soc. Den., 33:199-201.

Sigal, J., 1977. Essai de zonation du Crétacé méditerranéen à l'aide des foraminiferes planctoniques. Geol. Mediterr., 4:99-108.

Sissingh, W., 1977. Biostratigraphy of Cretaceous nannoplankton. Geol. Mijnbouw, 56:37-50.

Svabenicka, L., 1991. Coccolithen-Stratigraphie der oeheren Oberkreide der Bohrung Metelen 1001 (Muensterlaender, NW-Deutschland). Facies, 24:107-111.

Thierstein, H.R., 1973. Lower Cretaceous calcareous nannoplankton biostratigraphy. Abh. Geol. Bundesanst. A., 29:1-52.

, 1976. Mesozoic calcareous nannoplankton biostratigraphy of marine sediments. Mar. Micropaleontol., 1:325-362.

Tornaghi, M.E., Premoli Silva, I., and Ripepe, M., 1989. Lithostratigraphy and planktonic foraminiferal biostratigraphy of the Aptian-Albian "Scisti a Fucoidi" in the Piobbico core, Marche, Italy: background for cyclostratigraphy. Riv. Ital. Paleontol. Stratigr., 95:223-264.

Date of initial receipt: 1 February 1994

Date of acceptance: 15 September 1994

Ms 144SR-071 